\title{
Structure of the hot object in the symbiotic prototype $Z$ Andromedae during its 2000-03 active phase ${ }^{\star}$
}

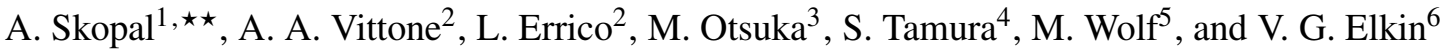 \\ 1 Astronomical Institute, Slovak Academy of Sciences, 05960 Tatranská Lomnica, Slovakia \\ 2 INAF Osservatorio Astronomico di Capodimonte, via Moiariello 16, 80131 Napoli, Italy \\ Okayama Astrophysical Observatory, NAOJ, Kamogata, Okayama 719-0232, Japan \\ 4 Astronomical Institute, Tohoku University, Sendai 980-8578, Japan \\ Astronomical Institute, Charles University Prague, 18000 Praha 8, V Holešovičkách 2, Czech Republic \\ ${ }^{6}$ Centre for Astrophysics, University of Central Lancashire, Preston PR1 2HE, UK
}

Received 22 February 2005 / Accepted 2 March 2006

\section{ABSTRACT}

\begin{abstract}
Aims. To investigate structure of the hot object in the symbiotic prototype Z And during its major 2000-03 active phase. Methods. Analysis of the far ultraviolet, optical low- and high-resolution spectroscopy and UBVR photometry. Reconstruction of the spectral energy distribution (SED) during the outburst. The Raman scattering process.

Results. At the initial stages of the outburst the hot object was characterized by the two-temperature spectrum (a warm stellar radiation and a strong nebular emission) with signatures of a mass-outflow at moderate $\left(\sim 100-200 \mathrm{~km} \mathrm{~s}^{-1}\right)$ and very high $\left(\approx 1000-2000 \mathrm{~km} \mathrm{~s}^{-1}\right)$ velocities. The corresponding structure of the hot object consists of an optically thick, slowly-expanding disk-like material encompassing the accretor at the orbital plane and a fast optically thin wind over the remainder of the star. The disk-like shell persisted around the central star until 2002 August as was indicated by the eclipse effect. Then, a significant dilution of the optically thick material and evolution of a fast wind from the hot star, concentrated more at the orbital plane, were detected. A striking similarity of [Fe VII] $\lambda 6087$ and Raman $\lambda 6825$ profiles at/after the dilution of the disk suggests their origin within the interaction zone where the winds from the binary components collide.
\end{abstract}

Key words. stars: activity - stars: binaries: symbiotic - stars: individual: Z And - stars: winds, outflows

\section{Introduction}

$\mathrm{Z}$ And is considered a prototype of the class of symbiotic stars. The binary is composed of a late-type, M4.5 III, giant and a white dwarf accreting from the giant's wind on a 758-day orbit (e.g. Nussbaumer \& Vogel 1989; Mikolajewska \& Kenyon 1996). More than 100 years of monitoring Z And (first records from 1887) demonstrated an eruptive character of its light curve (LC). It displays several active phases during which fluctuations ranges in amplitude from a few tenths of a magnitude to about 3 mag. During the strongest outbursts $(1915,1940$, $1960,1985,2000)$ the star's brightness reached values around $m_{\mathrm{pg}} \sim 9$ and $m_{\mathrm{vis}} \sim 8.5 \mathrm{mag}$ with a recurrence time of 15 to 25 years (see Fig. 1). Major outbursts are characterized by a decrease in the effective temperature of the hot object and the fluxes of some high ionization emission lines. Analyzing previous large eruptions, Mikolajewska \& Kenyon (1996) found that an A-F absorption spectrum characterized the 1940 optical maximum and during the 1960 outburst the system resembled an F0-2 supergiant. They estimated a roughly constant luminosity and a factor of 10 lower temperature, $T_{\mathrm{h}}$, for the hot object during eruptions with respect to quantities from quiescence. For the 1985 eruption, Mikolajewska \& Kenyon (1996) supported the decrease in $T_{\mathrm{h}}$ also by a decline of Raman scattered O VI lines

\footnotetext{
* Appendices are only available in electronic form at http://www. edpsciences.org

$\star \star$ Visiting Astronomer: Capodimonte Astrophysical Observatory.
}

at $\lambda 6825$ and $\lambda 7088$. This outburst was studied in detail by Fernández-Castro et al. (1995). Ultraviolet spectroscopy and radio observations indicated a considerably cooler UV continuum with an additional attenuation below $1300 \AA$ accompanied by a decline in the radio flux during the 1985 optical maximum. In the line spectrum they measured a broadening of all emission lines with signatures of P-Cygni type profiles for strong resonance lines. Fernández-Castro et al. (1995) interpreted these features as a result of the ejection of an optically thick shell at a moderately high velocity of $200-300 \mathrm{~km} \mathrm{~s}^{-1}$. Radio observations also provided evidence of mass outflow from the active star. At the initial stage of both the 1985 and 2000 outbursts the radio flux dropped below values from quiescence, then recovered along the optical decline and reached close to the quiescent level or above it (Fernández-Castro et al. 1995; Brocksopp et al. 2004). For the 1984-85 activity, Fernández-Castro et al. (1995) explained the anticorrelation between the visual and far-UV LCs by the extinction of UV radiation from the central source by a false photosphere produced by the ejection of a shell of dense material. They compared the subsequent rise of the radio emission with a model in which the ionizing source is turned on instantaneously at the center and an ionization front propagates out through the wind from the hot star. For the 2000-03 active phase, Brocksopp et al. (2004) revealed a jet-like extension at $5 \mathrm{GHz}$ on the 2001 September MERLIN image. They associated this transient feature with a blob of matter ejected at the beginning 
of the optical outburst, emitting most probably through thermal bremsstrahlung of ionized hydrogen gas.

On 2000 September 1st Z And entered a major outburst (Skopal et al. 2000). It reached a maximum brightness around mid 2000 December ( $U \sim 8.4, B \sim 9.3$ and $V \sim 8.8 \mathrm{mag}$ ) with a gradual decrease to quiescent values at/after mid 2003 (Skopal et al. 2004). In the summer of 2002, Skopal (2003a) observed a narrow minimum in the $\mathrm{LC}$ at the position of the inferior conjunction of the giant. He associated this effect with the eclipse of the active object by the giant and determined the orbital inclination of the system as $\gtrsim 76^{\circ}$. Sokoloski et al. (2006) observed the 2000-03 outburst in the X-ray, far ultraviolet, optical and radio. They focused on the nature of the outburst, and described observational evidence for a new kind of outburst that involves both an accretion-disk instability and a thermonuclear shell burning on the surface of an accreting white dwarf.

In this paper we present and analyze our original spectroscopic and photometric observations complemented by the archival spectra obtained with the Far Ultraviolet Spectroscopic Explorer (FUS E) during the 2000-03 major outburst of Z And. In Sect. 3 we analyze the data. We model the SED (Sect. 3.3) and examine the Raman scattering process (Sect. 3.4). In Sect. 4 we summarize the main results to reconstruct the basic structure of the hot object and the surrounding environment during the outburst.

\section{Observations and reductions}

\subsection{Optical spectroscopy}

Our spectroscopic observations were made during the recent major 2000-03 active phase at different observatories.

Prior to the maximum of the star's brightness, low-dispersion spectroscopy was secured at the Special Astrophysical Observatory of the Russian Academy of Sciences (SAO in Table 1) with the long slit UAGS spectrograph equipped with a fast Schmidt camera and CCD detector $(530 \times 580$ pixels of $18 \times 24 \mu \mathrm{m}$ size) mounted at the Cassegrain focus of the Zeiss 1-m telescope. The linear dispersion was 1.5 and $3.1 \AA$ pixel $^{-1}$ in the blue and red part of the spectrum, respectively. Basic reduction including extraction of 1D-spectra from CCD images and wavelength calibration were made using the software-package of Vlasyuk (1993).

At the maximum of the star's brightness, high-dispersion spectroscopy was secured at the Asiago Astrophysical Observatory (OAC) with the REOSC Echelle Spectrograph (RES) equipped with a Thompson THX31156 UV-coated CCD detector $(1024 \times 1024$ pixels of $19 \mu \mathrm{m}$ size $)$ mounted at the Cassegrain focus of the 1.82-m telescope at Mt. Ekar. Two very different exposures of 120 and $3600 \mathrm{~s}$ were applied to obtain a well defined $\mathrm{H} \alpha$ profile and the underlying continuum. The RES orders were straightened through the software developed at the Astronomical Observatory of Capodimonte in Napoli. Thereafter, the spectroscopic data were processed with the MIDAS software package. The dispersion of the RES was $10 \AA \mathrm{mm}^{-1}\left(0.19 \AA\right.$ pixel $\left.^{-1}\right)$ at $\mathrm{H} \alpha$. The spectra obtained during each night cover the wavelength range of $459.5-909.2 \mathrm{~nm}$.

After the maximum and the following transition to quiescence, high-dispersion spectroscopy was secured at the Okayama Astrophysical Observatory (OAO) with the HIgh Dispersion Echelle Spectrograph (HIDES, Izumiura 1999) at the $\mathrm{f} / 29$ Coudé focus of the $1.88-\mathrm{m}$ telescope. The dimension of the CCD (EEV 42-80) was $2048 \times 4096$ pixels of $13.5 \mu \mathrm{m}^{2}$.
Table 1. Log of spectroscopic observations.

\begin{tabular}{ccccc}
\hline \hline Date & $\begin{array}{c}\text { Julian date } \\
\text { JD } 24 \ldots\end{array}$ & Phase $^{\star}$ & $\begin{array}{c}\text { Region } \\
{[\mathrm{nm}]}\end{array}$ & Observatory \\
\hline $02 / 11 / 00$ & 51851.27 & 0.143 & $409.2-494.8$ & SAO \\
$04 / 11 / 00$ & 51853.34 & 0.146 & $413.2-495.0$ & SAO \\
$05 / 11 / 00$ & 51854.37 & 0.147 & $413.8-495.0$ & SAO \\
$05 / 11 / 00$ & 51854.39 & 0.147 & $482.0-563.1$ & SAO \\
$16 / 11 / 00$ & 51865.00 & 0.161 & $90.5-118.8$ & FUSE \\
$27 / 11 / 00$ & 51876.00 & 0.176 & $90.5-118.8$ & FUSE \\
$10 / 12 / 00$ & 51889.41 & 0.194 & $459.5-909.2$ & Asiago \\
$11 / 12 / 00$ & 51890.35 & 0.195 & $459.5-909.2$ & Asiago \\
$12 / 12 / 00$ & 51891.30 & 0.196 & $459.5-909.2$ & Asiago \\
$15 / 12 / 00$ & 51894.00 & 0.200 & $90.5-118.8$ & FUSE \\
$05 / 01 / 01$ & 51914.92 & 0.227 & $580.0-700.0$ & OAO \\
$08 / 01 / 01$ & 51917.90 & 0.231 & $450.0-570.0$ & OAO \\
$27 / 05 / 01$ & 52057.26 & 0.415 & $600.0-700.0$ & OAO \\
$22 / 07 / 01$ & 52113.30 & 0.489 & $90.5-118.8$ & FUSE \\
$30 / 09 / 01$ & 52183.30 & 0.582 & $90.5-118.8$ & FUSE \\
$10 / 02 / 02$ & 52315.93 & 0.757 & $580.0-700.0$ & OAO \\
$05 / 07 / 02$ & 52461.50 & 0.949 & $90.5-118.8$ & FUSE \\
$07 / 08 / 02$ & 52494.25 & 0.992 & $580.0-700.0$ & OAO \\
$22 / 10 / 02$ & 52570.50 & 0.093 & $90.5-118.8$ & FUSE \\
$02 / 02 / 03$ & 52673.45 & 0.229 & $580.0-700.0$ & OAO \\
$31 / 07 / 03$ & 52852.50 & 0.465 & $580.0-700.0$ & OAO \\
$04 / 08 / 03$ & 52856.80 & 0.471 & $90.5-118.8$ & FUSE \\
$02 / 09 / 03$ & 52885.50 & 0.509 & $580.0-700.0$ & OAO \\
$13 / 11 / 03$ & 52957.43 & 0.604 & $625.8-677.0$ & Ondřejov \\
$18 / 12 / 03$ & 52992.28 & 0.650 & $625.8-677.0$ & Ondřejov \\
\hline JD 1 Min $=2414625.2+757.5 \times E(S k o p a l 1998)$. & \\
& & & &
\end{tabular}

The spectral resolving power was 50000 or 68000 . The dispersion was $\sim 0.02 \AA \mathrm{pixel}^{-1}\left(\sim 1.2 \mathrm{~km} \mathrm{~s}^{-1}\right)$ and $\sim 0.03 \AA \mathrm{pixel}^{-1}$ $\left(\sim 1.4 \mathrm{~km} \mathrm{~s}^{-1}\right)$ in the regions of $450-570$ and $580-700 \mathrm{~nm}$, respectively. Bias and instrumental flats were also measured and taken into account in the usual way. The reduction and analysis was performed with the IRAF-package software and our own code to reduce spectra obtained with HIDES. For example, the scattered light from the instrument was also removed. The Th-Ar comparison spectrum was fitted with a 3 rd-order polynomial function, which reproduced the wavelength calibration to better than $10^{-3} \AA$.

At the post-outburst stage (the end of 2003) high-resolution spectroscopy between 6258 and $6770 \AA$ was performed at the Ondřejov Observatory using the coude spectrograph of $2-\mathrm{m}$ reflector and the BROR CCD camera with the SITe $800 \times$ 2030 pixels chip. The resolution power at the $\mathrm{H} \alpha$ region was 13000 . Basic treatment of the spectra was done using the IRAF-package software.

A correction for heliocentric velocity was applied to all spectra. The journal of spectroscopic observations is given in Table 1.

\subsubsection{Flux calibration of the spectra}

We converted arbitrary flux units of our spectra to fluxes in erg cm $\mathrm{cm}^{-2} \mathrm{~s}^{-1} \AA^{-1}$ with the aid of the simultaneous $U B V R$ photometry. (i) We summarized photometric observations carried out (nearly-)simultaneously with the spectrum under consideration. (ii) We calculated corrections $\Delta m$, which are due to emission lines in the spectrum, to obtain magnitudes corresponding to the continuum (see Skopal 2003b, for details). (iii) We dereddened the continuum-magnitudes with $E_{B-V}=0.30$ by using the extinction curve of Cardelli et al. (1989) and converted 
to fluxes according to the calibration of Henden \& Kaitchuck (1982). (iv) We determined the star's continuum by fitting the $U B V R$ fluxes with a 3-order polynomial function and scaled the measured spectrum to this fit.

\subsection{Far ultraviolet spectroscopy}

The FUSE instrument is described in detail by Moos et al. (2000) and Sahnow et al. (2000). A brief useful description of the spectrograph is given in Young et al. (2005).

The data were processed by the calibration pipeline version 3.0.7, 3.0.8 and 2.4.1. We used the calibrated time-tag observations (TTAG photon collecting mode) taken through the large aperture (LWRS). Before adding the flux from all exposures we applied an appropriate wavelength shift relative to one to obtain the best overlap of the absorption features. Then we co-added spectra of individual exposures and weighted them according to their exposure times. We binned the resulting spectrum within $0.025 \AA$. In order to determine the wavelength scale of the spectra, a correction for heliocentric velocity including that of the satellite was applied. We then selected interstellar absorption lines for which wavelengths are known and fitted the corresponding observed features. For example, in the region of the LiF1A spectra we used N I $\lambda 1003.372$, Si II $\lambda 1020.699$, A I $\lambda 1048.218$ (e.g. Rogerson \& Ewell 1984, Fig. 3 here). The accuracy of such calibration is of $\pm 0.05 \AA$. The data retrieved from the FUSE archive were processed mostly by own codes (AS).

Dereddening the FUSE spectra we found the extinction curve of Cardelli et al. (1989) to be valid only in the interval from the long-wavelength end of the spectra to about $1100 \AA$ (open circles in Figs. 6 and 7; $E_{B-V}=0.30, R_{V}=3.1$ ). Beyond these wavelengths to $1000 \AA$, dereddened fluxes are greater by a factor of 1.5-2 with respect to quantities required by our modeling of the SED (crosses in Fig. 7). Note that the extinction curve in the far-ultraviolet (1250-1000 ̊) as suggested by Cardelli et al. (1986, Fig. 5 there) represents only an extrapolation and thus is more uncertain. As a result we used their mean extinction law only for $\lambda>1100 \AA$. The short-wavelength part of the spectrum was corrected to our model SED (Fig. 6).

\subsection{Broad-band photometry}

Photometric observations were performed in the standard Johnson $U B V$ system with the $R_{\mathrm{C}}$ filter of the Cousins system. We used single-channel photoelectric photometers mounted in the Cassegrain foci of $0.6-\mathrm{m}$ reflectors at the Skalnate Pleso and Stará Lesná observatories. We converted our $R_{\mathrm{C}}$ magnitude to that of the Johnson system using transformations of Bessell (1983). Z And was measured relative to the comparison star SAO 53150 (BD+47 4192; $V=8.99, B-V=0.41$, $U-B=0.14, V-R=0.16$ ). Usually, a 1-hour cycle contained about 10 to 20 individual differences between the target and the comparison. This approach reduced the internal uncertainty to $\sim 0.01 \mathrm{mag}$ in the $V, B$ and $R$ bands, and up to $0.025 \mathrm{mag}$ in the $U$ band. Individual measurements were published by Skopal et al. (2004).

In this paper we dereddened our observations with $E_{B-V}=$ 0.30 and scaled relevant parameters for a distance of $1.5 \mathrm{kpc}$ (Skopal 2005, and references therein).

\section{Analysis}

\subsection{Photometric evolution}

The bottom panels of Fig. 1 show the UBVR LCs of Z And covering its recent outburst. The rise to maximum was characterized by three rapid increases in the star's brightness and two plateaus, best seen in the $U$ band. The initial rise between 2000 September 1 st and 7 th $(\Delta U \doteq 1.9, \Delta B \doteq 1.5$, $\Delta V \doteq 1.1)$ was probably caused by an increase in the hot object luminosity. The $U-B$ index became more blue with respect to observations made prior to the outburst. Consequently, the profile of the optical continuum became more steep. Its nature nebular or stellar (or a combination of both) - is important to understand the trigger mechanism of the outburst. The nebular nature of the optical light at the beginning of the outburst would require an increase in $T_{\mathrm{h}}$ to provide the necessary amount of ionizing photons, while the stellar nature of the optical radiation would be associated with the creation of a shell surrounding the hot star, thus implying a decrease in $T_{\mathrm{h}}$. Unfortunately, we do not have relevant spectroscopic observations from the initial stage of the activity to distinguish between these possibilities. Nonetheless a comparison of colour indices with those of a black body (they are well above blackbody values, see Fig. 1 of Skopal 2003b) signals a contribution from other sources. The following short-term decline in the $U-B$ index at constant $B-V$ and $V-R$ indices suggests a decrease of the hot component temperature. During the "totality" of the $U-B$ minimum ( JD $2451800-2451865)$ all the colour indices were practically constant indicating a constant temperature. Therefore the second increase in the star's brightness, which occurred at constant indices ( JD 2451820 , Fig. 1), had to result from an increase in the luminosity at a relevant expansion of the shell. The third brightening from mid-November to the maximum (i.e. the ascending branch of the $U-B$ minimum) indicated an increase of the hot object temperature and its luminosity. Quantities of the corresponding colour indices were probably due to a composition of contributions from nebula and stellar sources. Our SED model from the period of maximum supports this interpretation (Sect. 3.3.2, Fig. 7). A similar behaviour in colour indices was also observed at early stages of classical novae, prior to the maximum (e.g. Fig. 3 of Chochol \& Pribulla 1997).

From $2001 \mathrm{Z}$ And entered the decline phase during which both colour indices and the star's brightness were evolving toward quantities of the quiescent phase at the end of 2003 (Fig. 1). In August of 2002 the gradual decline was interrupted by the eclipse effect. This event revealed a high orbital inclination and the profile of the minimum suggested a disk-like structure for the eclipsed object (see Skopal 2003a, in detail). In addition, a transient $\sim 0.6 \mathrm{mag}$ rebrightening lasting from October 2002 to March 2003 was indicated in the LC (see Sect. 3.6 in more detail).

\subsection{Spectroscopic evolution}

Figures 2-6 show the main characteristics of our spectroscopic observations along the outburst, from its maximum in 2000 December to its end in 2003. We comment on them as follows:

(i) The P-Cygni profiles of H I lines of the Paschen series and He I lines were present mainly at the initial stages of the outburst and at its maximum. The main absorption component was located at about $-90 \mathrm{~km} \mathrm{~s}^{-1}$. However, complex absorption features of up to $\sim-300 \mathrm{~km} \mathrm{~s}^{-1}$ could be recognized at $\lambda 8502$, 

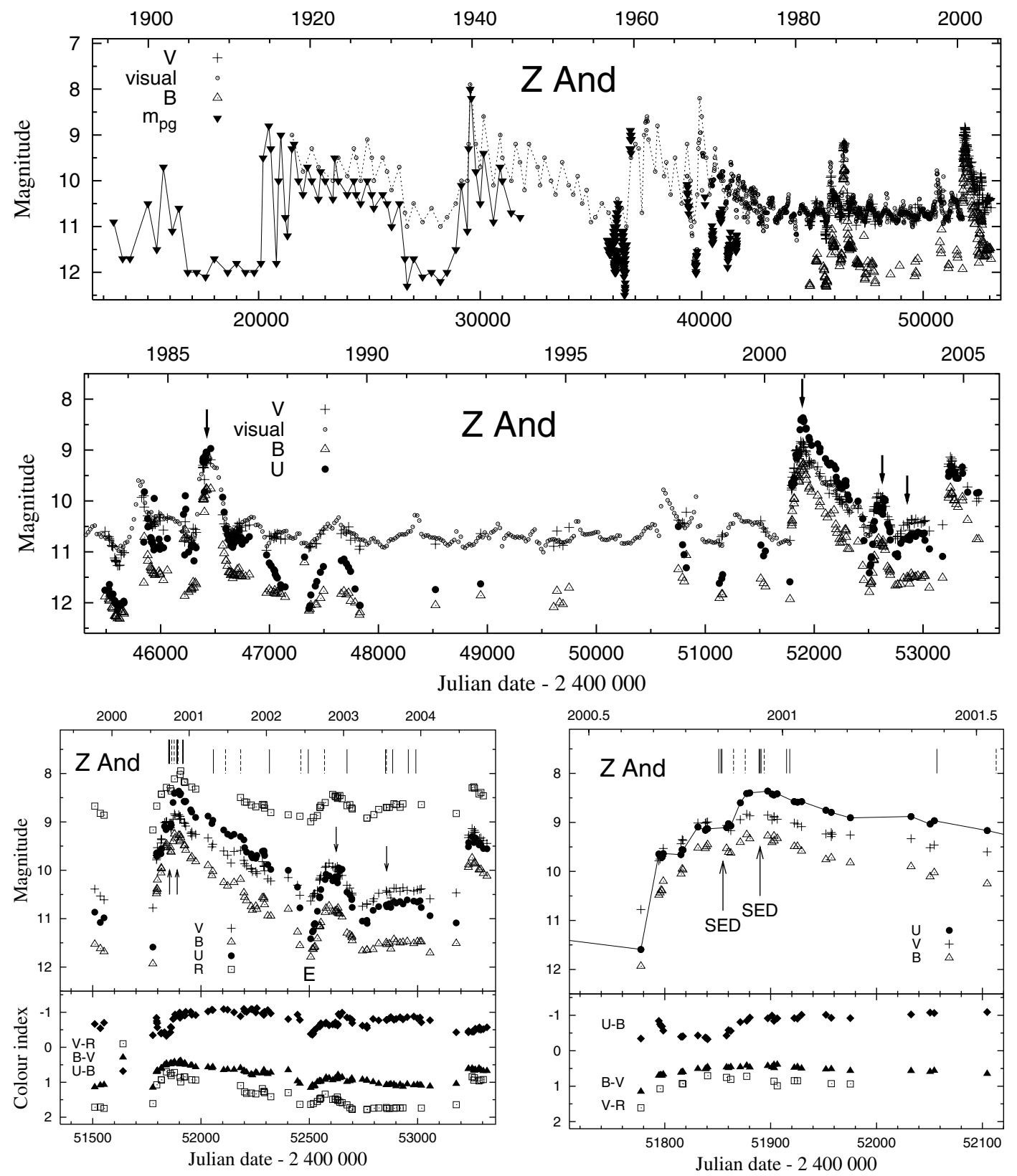

Fig. 1. Top: the historical 1895-2004 photographic/B-band/visual LC of Z And. It is compiled from the data published by Payne-Gaposchkin (1946), Romano (1960), Mjalkovskij (1977), Belyakina (1985, 1992), Mikolajewska \& Kenyon (1996), Skopal et al. (2002b, 2004) and smoothed visual AFOEV data. Middle: a part of the multicolour LC covering the recent two major outbursts (1984-86 and 2000-03). Bottom: the UBVR photometry from the major 2000-03 outburst. Left panel shows the overall evolution in the LC. The eclipse is denoted by E. The right panel shows the LC at the initial stage of the outburst - note its stepped profile on the rise to the maximum. Optical/FUV spectroscopic observations are denoted by vertical full/dashed bars. The arrows mark positions at which we reconstructed the observed SED.

$\lambda 8545$ and $\lambda 8665$. In the far-UV Sokoloski et al. (2006) detected P-Cygni profiles in C III and P V lines (Fig. 6 here) with terminal velocities of $\approx-200 \mathrm{~km} \mathrm{~s}^{-1}$ in the first FUSE observations. They also demonstrated the evolution from an absorption to emission profile of these lines as the outburst progressed to its maximum. On the other hand, the resonance line O VI $\lambda 1032$ shows a strong absorption in the P-Cygni line profile throughout the whole active phase (Fig. 3). This suggests its origin in the stellar wind of the hot star. During quiescence the absorption component was not present in the profile (Schmid et al. 1999). In Sect. 3.4 we examine this line in detail.

(ii) The $\mathrm{H} \beta$ wings extended to about $1000 \mathrm{~km} \mathrm{~s}^{-1}$ or more. The main emission core showed an asymmetrical profile with the peak placed at about $+30 \mathrm{~km} \mathrm{~s}^{-1}$ (the systemic velocity is $-1.8 \mathrm{~km} \mathrm{~s}^{-1}$ ). The asymmetry can result from superposition of a violet shifted absorption component created in the neutral gas at the front of the expanding material. The $\mathrm{H} \alpha$ profile exhibited similar behaviour. However, its wings extended to more than $\pm 2000 \mathrm{~km} \mathrm{~s}^{-1}$ and the asymmetry was more pronounced. At/after the spectroscopic conjunction the absorption component created a cut-out on the violet side of the wing at about $-40 \mathrm{~km} \mathrm{~s}^{-1}(07 / 08 / 02)$ and $-50 \mathrm{~km} \mathrm{~s}^{-1}(02 / 02 / 03)$. During the low-amplitude brightening (2002/03) we measured an additional extension of the $\mathrm{H} \alpha$ wings on our spectrum from $02 / 02 / 03$. We ascribe their origin to the stellar wind from the hot star (Sect. 3.5). 

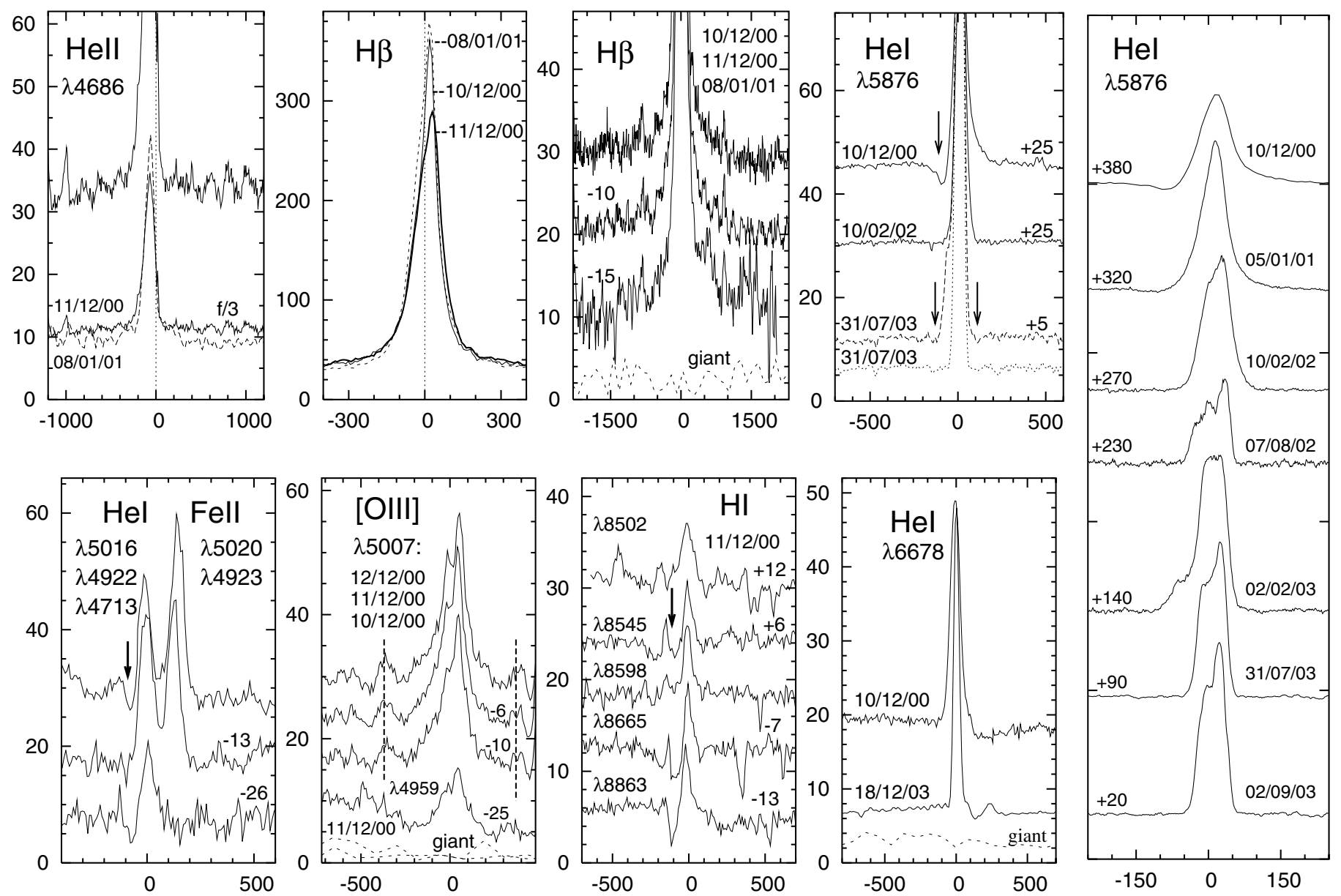

Fig. 2. Evolution in selected lines, mainly from the optical maximum. Fluxes are in units of $10^{-13} \mathrm{erg} \mathrm{cm}^{-2} \mathrm{~s}^{-1} \AA^{-1}$ and radial velocities in $\mathrm{km} \mathrm{s} \mathrm{s}^{-1}$. Small numbers in panels with more profiles represent their shift with respect to the level of the local continuum. The arrows denote absorption components. The right panel shows evolution in the He I $\lambda 5876$ line ( $y$-tics are by 100 units). The continuum from the giant, as given by the SED (Fig. 7), is compared to clarify details in lines of $\mathrm{H} \beta$, [O III] $\lambda 5007$ and $\mathrm{He} \mathrm{I} \lambda 6678$.

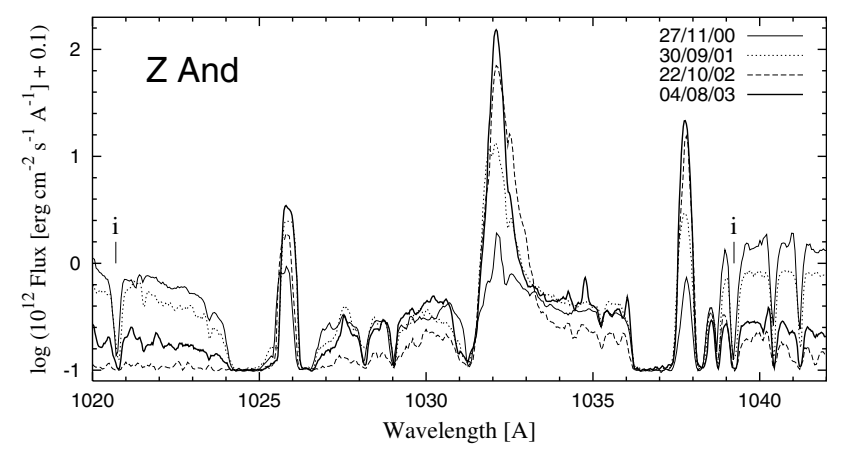

Fig. 3. Evolution in the O vi $\lambda 1032$ line (LiF1A spectra) in an appropriate logarithmic scale. Note that the P-Cygni profile with a strong absorption developed throughout the whole active phase. Positions of interstellar lines are denoted by $\mathbf{i}$.

(iii) At the maximum, the main emission core of the He II $\lambda 4686$ was shifted by about $-75 \mathrm{~km} \mathrm{~s}^{-1}$ at the peak and by about $-105 \mathrm{~km} \mathrm{~s}^{-1}$ at its bottom with respect to the reference wavelength. The [Fe VII] $\lambda 6087$ line displayed similar characteristics. Its profile was very low and broad with the main emission located around $-80 \mathrm{~km} \mathrm{~s}^{-1}$ (Fig. 4). At the transition from the optical maximum to quiescence these lines became stronger, being located at/around their reference wavelengths (see Fig. 4 for [Fe VII] and Tomov et al. 2005 for the He II line). The evolution in these lines is discussed in Sect. 4.

(iv) He I $\lambda 5876, \lambda 6678$ and $\lambda 7065$ emission lines can be used to diagnose symbiotic nebulae (Proga et al. 1994). We observed all these lines at the maximum, but later, along the investigated active phase, only $\lambda 5876$ and $\lambda 6678$ were measured. At the beginning of the outburst they developed a P-Cygni type of profile. From 2002 August absorptions affected the whole profile, mainly that of the $\lambda 5876$ line (Fig. 2). A narrow emission core without any wings and with absorption features at the top of the profile were observed. The ratios of their fluxes differ significantly from those arising from net recombination. For example, we measured the ratio $\Lambda=F(5876) / F(6678) \sim$ 1.2-1.5, while theoretical intensities from low-density plasma predict $\Lambda \sim 3.6$ (e.g. Smits 1996). This difference has been ascribed to collisions and optical depth due to the metastability of the $2{ }^{3} \mathrm{~S}$ level, which affect the He I level populations in high density plasma (e.g. Clegg 1987; Schmid 1989a; Proga et al. 1994; Kingdon \& Ferland 1995). Therefore we compared our flux ratios, $F(6678) / F(5876)$ and $F(7065) / F(5876)$ with those modeled by Proga et al. (1994) for the case of high density nebulae in symbiotic stars (Appendix A, Fig. A.1). This suggests that the $\mathrm{He}+$ region is very compact with an average 

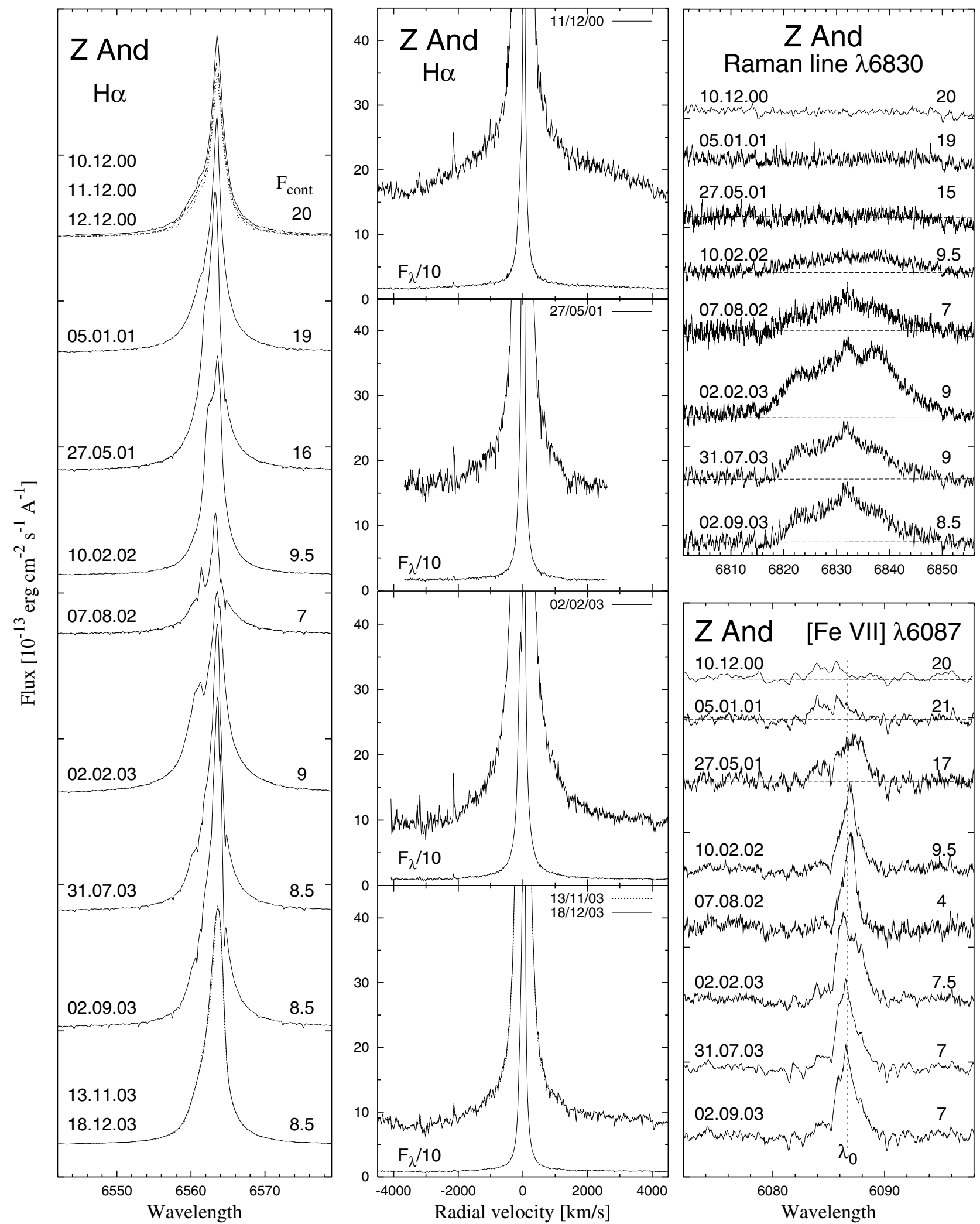

\section{Z And $[\mathrm{Fe} \mathrm{VII]} \lambda 6087$}

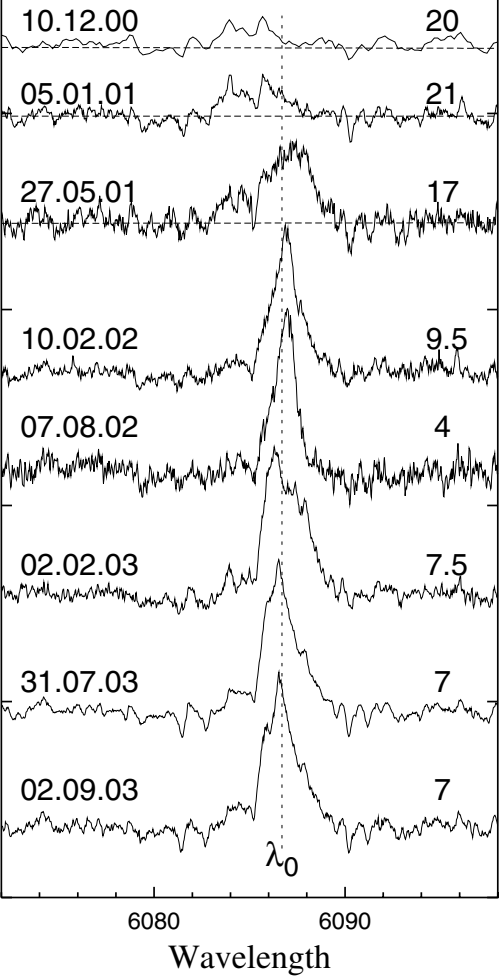

Fig. 4. Evolution in the $\mathrm{H} \alpha$, Raman $\lambda 6825$ and [Fe VII] 6087 line profiles along the outburst, from the 2000-maximum to 2003-quiescence. Left panel shows profiles of the $\mathrm{H} \alpha$ line core ( $y$-tics are by 500 units), while the middle panels show the extended wings of the profiles in detail. On the right panels $y$-intervals are of 20 units and the horizontal dashed lines represent the approximated continuum as described in Sect. 2.1.1.

density $N_{\mathrm{e}} \approx 10^{12} \mathrm{~cm}^{-3}$, radiating at $T_{\mathrm{e}} \approx 20000 \mathrm{~K}$. Emissivity of such plasma in the HeI $\lambda 5876$ line corresponds to an effective radius, $R_{\mathrm{eff}}(\mathrm{He}+) \approx 5 R_{\odot}$, which represents the radius of a sphere with constant density (see Appendix A in more detail). (v) Profile of the nebular [O III] $\lambda 5007$ line showed asymmetrically extended core/wings with terminal velocities of about -300 and $+250 \mathrm{~km} \mathrm{~s}^{-1}$. A sequence of profiles from 2000 December 10, 11 and 12th, showed the creation of an extra 


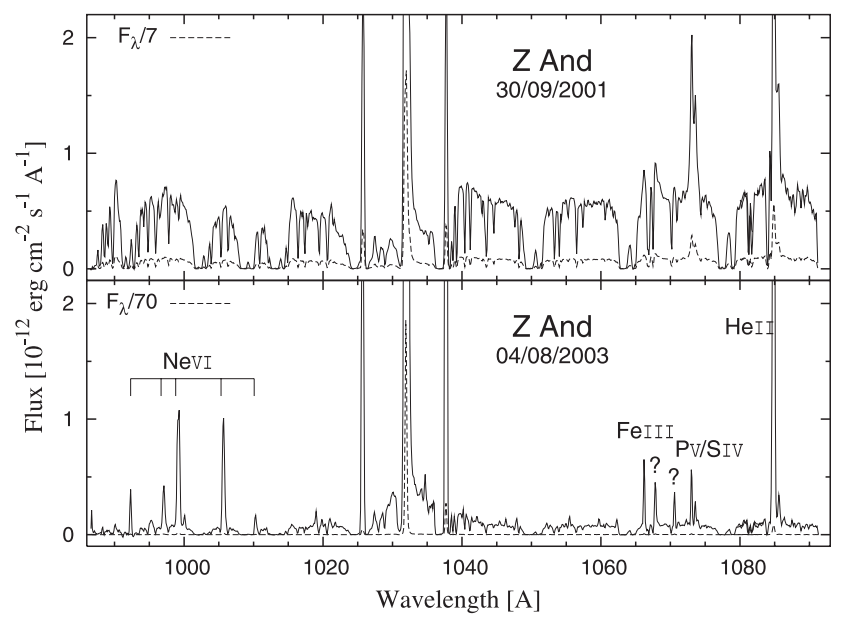

Fig. 5. Observed LiF1A spectra from 30/09/01 and 04/08/03. The level of the continuum significantly decreased while fluxes of the O VI doublet increased by a factor of $\sim 10$ on $04 / 08 / 03$. Note also the appearance of the Ne VI lines.

emission component on the violet side of the main core. In addition, weak emission components at about $\pm 380 \mathrm{~km} \mathrm{~s}^{-1}$ accompanied the main core during the maximum. Their reality is supported by the $\mathrm{S} / \mathrm{N}$ ratio of about 10 at these wavelengths and a relatively low level of the continuum from the giant (cf. Figs. 2 and 7). If one associates these components with polar blobs, as was interpreted for the classical Nova V 1974 by Chochol et al. (1997), the space velocity of the ejecta would be larger than $\sim 1600 \mathrm{~km} \mathrm{~s}^{-1}$ for $i>76^{\circ}$ (Skopal 2003a).

(vi) Evolution in the Raman scattered O VI line at $\lambda 6825$ provides information on the ionization structure around the active object. This emission was absent on our spectra from the maximum and after it. It appeared first on the 10/02/02 spectrum as a very faint emission. During 2003 it became strong and broad $(F W Z I \approx 25-30 \AA$ ) with $1-3$ knots at the top of its profile (Fig. 4). Schmid (1989b) suggested that this emission feature is due to Raman scattering of the O VI $\lambda 1032$ resonance line by atoms of neutral hydrogen. In Sect. 3.4 we investigate this process in detail.

(vii) Transition to quiescence was characterized mainly by a significant increase in the hot object temperature (Sect. 3.3.3). As a result fluxes of highly ionized elements increased and new ones appeared (e.g. Ne VI lines, Fig. 5). At the end of 2003 a constant brightness near the values from quiescence and very stable $\mathrm{H} \alpha$ profiles (13/11/2003 and 18/12/2003) suggested the $\mathrm{Z}$ And active phase to be over. However, a strong wind from the hot star was still indicated by the P-Cygni profiles of the O VI line (Fig. 3), and in 2004 August we detected a new eruption in the LC (Fig. 1).

\subsection{Spectral energy distribution}

Here we aim to reconstruct the SED prior to the maximum, at the maximum and during the post-outburst stage to better understand the observed photometric and spectroscopic evolution throughout the outburst.

\subsubsection{Model of the composite spectrum}

According to a simple photoionization model for symbiotic stars proposed by Seaquist et al. (1984) (hereafter STB), the observed flux in the continuum, $F(\lambda)$, can be expressed as

$F(\lambda)=F_{\mathrm{g}}(\lambda)+F_{\mathrm{h}}(\lambda)+F_{\mathrm{N}}(\lambda)$

where $F_{\mathrm{N}}(\lambda)$ is the nebular component of radiation and $F_{\mathrm{h}}(\lambda)$ and $F_{\mathrm{g}}(\lambda)$ are stellar components from the hot star and the cool giant, respectively. We approximated radiation from the giant by a synthetic spectrum, $F_{\lambda}^{\text {synth. }}\left(T_{\text {eff }}\right)$ (models from Hauschildt et al. 1999), the hot stellar continuum by a black body at the temperature $T_{\mathrm{h}}$ including the effect of Rayleigh scattering and the nebular radiation in the continuum by processes of recombination and thermal bremsstrahlung in the hydrogen plasma. Then Eq. (1) takes the form (Skopal 2005, Eq. (13))

$F_{\lambda}=F_{\lambda}^{\text {synth. }}\left(T_{\text {eff }}\right)+\theta_{\mathrm{h}}^{2} \pi B_{\lambda}\left(T_{\mathrm{h}}\right) e^{-n_{\mathrm{H}} \sigma_{\lambda}^{\mathrm{R}}}+\frac{E M}{4 \pi d^{2}} \varepsilon_{\lambda}\left(\mathrm{H}, T_{\mathrm{e}}\right)$,

where $\theta_{\mathrm{h}}=R_{\mathrm{h}}^{\mathrm{eff}} / d$ is the angular radius of the hot stellar source given by its effective radius $R_{\mathrm{h}}^{\text {eff }}$ and the distance $d$. Parameters determining the Rayleigh attenuation are the column density of $\mathrm{H}$ atoms, $n_{\mathrm{H}}\left[\mathrm{cm}^{-2}\right]$, and the Rayleigh scattering cross-section for atomic hydrogen, $\sigma_{\lambda}^{\mathrm{R}}\left[\mathrm{cm}^{2}\right]$. Nebular radiation is in our approximation defined by the emission measure $E M$ and the volume emission coefficient for hydrogen $\varepsilon_{\lambda}\left(\mathrm{H}, T_{\mathrm{e}}\right) \quad\left[\mathrm{erg} \mathrm{cm}^{3} \mathrm{~s}^{-1} \AA^{-1}\right]$, which depends on the electron temperature $T_{\mathrm{e}}$. The method of disentangling the composite spectra of symbiotic stars was recently described by Skopal (2005).

To reconstruct the continuum of the hot object we used only the flux-points of the $U B V R$ photometry and the far-UV continuum from $\lambda 1188$ to $\lambda 1100 \AA$. With the aid of the FUSE spectra we determined the column density of the atomic hydrogen by fitting the Rayleigh attenuated hot stellar radiation to the observed continuum blueward Ly $\beta$ and Ly $\alpha$ lines (Fig. 6). This reduced free parameters in the model to $\theta_{\mathrm{h}}, T_{\mathrm{h}}, T_{\mathrm{e}}$ and $E M$. As there was no ultraviolet spectrum available from the 2000-03 outburst, determination of $T_{\mathrm{e}}$ is more uncertain. To satisfy the condition for an appropriate fit (Skopal 2005, Sect. 3.3) we estimated the uncertainty in $T_{\mathrm{h}}$ within the range of $500-1000 \mathrm{~K}$, while $T_{\mathrm{e}}$ can run between about 30000 and $40000 \mathrm{~K}$.

\subsubsection{SED at the plateau stage and the maximum}

To reconstruct the SED during the time of the last plateau in the LC we used the optical spectrum from 06/11/00 and the FUSE observation from 16/11/00. For the maximum, the corresponding data are from $11 / 12 / 00$ and $15 / 12 / 00$, respectively. A comparison of our solutions with observations is plotted in Fig. 7 and the corresponding parameters are given in Table 2 .

The change in the SED from the plateau at $U \sim 9.0$ to the maximum $(U \sim 8.3)$ was due to an increase in both the hot stellar and the nebular emission. These components of radiation contributed more to the $U$ band than to the $B V R$ region. Therefore we observed a larger increase of the star's brightness in $U(\Delta U \sim 0.7, \Delta B \sim 0.4, \Delta V \sim 0.3, \Delta R \sim 0.3 \mathrm{mag})$, i.e. the $U-B$ index became more blue. The temperature of the hot stellar source, $T_{\mathrm{h}}$, increased from about $26000 \mathrm{~K}$ to about $28000 \mathrm{~K}$ at a constant effective radius $R_{\mathrm{h}}^{\text {eff }}$ (Table 2$)$. Accordingly, its luminosity increased from 3800 to $5300(d / 1.5 \mathrm{kpc})^{2} L_{\odot}$ during this transition. However, the emission measure also increased significantly, by a factor of 2.2 . 


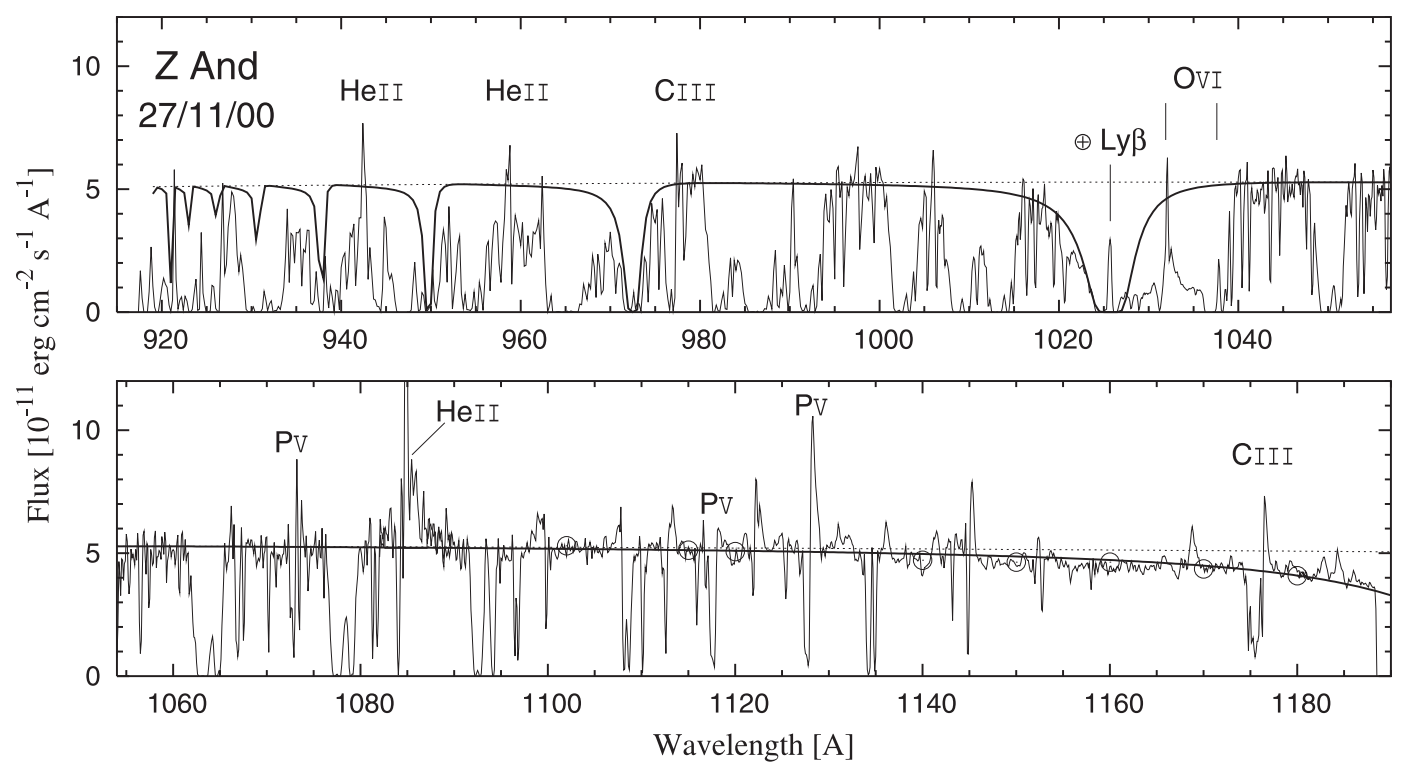

Fig. 6. Dereddened FUSE spectra of Z And from the maximum of the optical brightness. It is composed from $\mathrm{SiC} 2 \mathrm{~A}$ (917-1005 $\AA$ ), LiF1A (988-1082 ̊̊) and LiF2A (1087-1181 ̊̊) channel spectra. Spectra from SiC1A (1080-1090 $\mathrm{A})$ and LiF1B (1080-1188 $\mathrm{A})$ have been used to complete the wavelength coverage. The solid line represents the Rayleigh attenuated $\left(N_{\mathrm{H}}=7 \times 10^{21} \mathrm{~cm}^{-2}\right)$ blackbody radiation from the hot stellar source at $28000 \mathrm{~K}$ (Sect. 3.3.2), while the dotted line is not attenuated. Most of the absorption features are due to $\mathrm{H}_{2}$ in the interstellar medium (cf. Crowley et al. 2004). Note the P-Cygni type of profiles for C III, P V lines and the broad emission in the He II $\lambda 1084$ profile.

The observed hot stellar radiation is not capable of producing the nebular emission (too low $T_{\mathrm{h}}$ ). This implies that the hot ionizing source cannot be seen directly by the outer observer, but can be "seen" by the surrounding nebula, which converts its radiation into the nebular emission. This suggests a disk-like structure of the optically thick material, whose outer rim occults the central hot star in the direction of the observer (also Sect. 5.3.5 of Skopal 2005). For comparison we show the SED from the previous 1986 optical maximum, which is of the same type. In such a case the temperature of the ionizing source, $T_{\mathrm{h}}^{\text {i.s. }}$, can be determined only indirectly from the nebular radiation, which mirrors its radiative properties. For example, Sokoloski et al. (2006) determined $T_{\mathrm{h}}^{\text {i.s. }}=95000 \mathrm{~K}$ from fluxes of $\mathrm{H} \beta$ and He II 4686 emission lines observed at the maximum. However, scaling the hot radiation from the unseen ionizing source to the observed, significantly cooler, pseudophotosphere yields an erroneous (too high) luminosity of the white dwarf. According to our SED model the total luminosity produced at/around the white dwarf during the maximum is $L_{\mathrm{T}} \gtrsim L_{\mathrm{h}}+L_{\mathrm{N}}=$ $6900(d / 1.5 \mathrm{kpc})^{2} L_{\odot}$ (Table 2 here and Sect. 5.3.6 of Skopal 2005, in more detail), but Sokoloski et al. (2006) derived $L_{\mathrm{h}}=$ $9800(d / 1.2 \mathrm{kpc})^{2} L_{\odot}$ for $E_{B-V}=0.27\left(=21500(d / 1.5 \mathrm{kpc})^{2} L_{\odot}\right.$ for $\left.E_{B-V}=0.30\right)$ by scaling the $95000 \mathrm{~K}$ radiation of the ionizing source to the observed FUV fluxes from the warm pseudophotosphere. Also the best model SED of the FUV and optical continuum with the fixed stellar component radiating at $T_{\mathrm{h}}^{\text {i.s. }}=95000 \mathrm{~K}$ is not satisfactory $(\chi \doteq 56.2)$. For comparison, our models in the middle panels of Fig. 7 have $\chi \doteq 8.5$ and $\chi \doteq 13.6$, respectively.

\subsubsection{SED at the post-outburst stage}

Here we selected the last observations made by the FUSE on 04/08/03 and corresponding $U B V R$ photometric measurements. The slope of the $B, V$ and $R$ fluxes has an opposite trend to that from the maximum - the fluxes follow rather the SED of the giant, which dominates the spectrum from the $B$ band to longer wavelengths (Fig. 7). The $U$ flux-point does not obey this trend. It is higher, the ratio $F_{\mathrm{U}} / F_{\mathrm{B}} \sim 1.78$ (i.e. $U-B=-1.16$ ). This reflects a dominant contribution from the nebula in the near$\mathrm{UV} / \mathrm{U}$. As a result the radiation from the hot star in the optical had to be small relatively to other sources here. This suggests a high temperature, $T_{\mathrm{h}} \gtrsim 100000 \mathrm{~K}$. We adopted $T_{\mathrm{h}}=120000 \mathrm{~K}$ to satisfy the basic photo-ionization model of symbiotic stars (STB). At this temperature the hot star luminosity is just capable of producing the observed EM. From this point of view $T_{\mathrm{h}} \geq 120000 \mathrm{~K}$. Finally, we complemented contributions from the giant and the hot stellar source with a nebular contribution with $T_{\mathrm{e}}=25000+5000 /-7000 \mathrm{~K}$ and $E M=1.3 \times 10^{60} \mathrm{~cm}^{-3}$. Large uncertainties in $T_{\mathrm{e}}$ are given by the absence of ultraviolet data. Model parameters are in Table 2.

Sokoloski et al. (2006) determined $T_{\mathrm{h}}=(160000 \pm 35000) \mathrm{K}$ from emission line fluxes and $R_{\mathrm{h}}^{\mathrm{eff}}=(0.06 \pm 0.01) \times 1.47=$ $(0.088 \pm 0.015) R_{\odot}, L_{\mathrm{h}}=(2000 \pm 500) \times 2.20=(4400 \pm 1100) L_{\odot}$ from the $F U V$ continuum fluxes. The factors 1.47 and 2.20 convert their original quantities calculated for $E_{B-V}=0.27$ and $d=$ $1.2 \mathrm{kpc}$ to those corresponding to $E_{B-V}=0.30$ and $d=1.5 \mathrm{kpc}$ we adopted in this paper. In this case physical parameters derived from the SED (Table 2) are the same as those given by optical line fluxes in combination with the $F U V$ continuum fluxes. This reflects an optically thin regime in 2003 - in contrast to the situation from the optical maximum.

\subsubsection{SED as an indicator of the mass outflow}

The total emission measure of the nebular continuum at the maximum, $E M \sim 6 \times 10^{60} \mathrm{~cm}^{-3}$ (Table 2), was a factor of 5-10 larger than in quiescence (Nussbaumer \& Vogel 1989; Skopal 2005) and a factor of 3 larger than at the maximum of the 1985 eruption (Fernández-Castro et al. 1995). During quiescent phases the nebula originates mostly from ionization of the giant's wind. It is characterized by a large parameter $X>10$ in the STB model (e.g. Fernández-Castro et al. 1988; Skopal 2001), which corresponds to a very open nebula (i.e. in major part a 
Table 2. Fitting and derived parameters from the SED during the maximum of the 1985 and 2000 outburst.

\begin{tabular}{rcccccccc}
\hline \hline $\begin{array}{c}\text { Date } \\
\text { FUSE-Optical }\end{array}$ & $\begin{array}{c}T_{\mathrm{h}} \\
{[K]}\end{array}$ & $\theta_{\mathrm{h}}$ & $\begin{array}{c}R_{\mathrm{h}}^{\text {eff }} \\
{\left[R_{\odot}\right]}\end{array}$ & $\begin{array}{c}L_{\mathrm{h}} \\
{\left[L_{\odot}\right]}\end{array}$ & $\begin{array}{c}\log \left(n_{\mathrm{H}}\right) \\
{\left[\mathrm{cm}^{-2}\right]}\end{array}$ & $\begin{array}{c}T_{\mathrm{e}} \\
{[K]}\end{array}$ & $\begin{array}{c}E M \\
{\left[\mathrm{~cm}^{-3}\right]}\end{array}$ & $\begin{array}{c}L_{\mathrm{N}} \\
{\left[L_{\odot}\right]}\end{array}$ \\
\hline $24 / 12 / 85$ & 19000 & $3.4 \times 10^{-11}$ & 2.3 & 590 & 23.34 & $18000 / 40000$ & $2.1 \times 10^{60}$ & 425 \\
$16 / 11 / 00-06 / 11 / 00$ & 26000 & $4.6 \times 10^{-11}$ & 3.1 & 3800 & 21.85 & 37000 & $3.9 \times 10^{60}$ & 740 \\
$27 / 11 / 00-11 / 12 / 00$ & 28000 & $4.7 \times 10^{-11}$ & 3.1 & 5300 & 21.85 & 38000 & $8.8 \times 10^{60}$ & 1600 \\
$04 / 08 / 03-31 / 07 / 03$ & 120000 & $1.5 \times 10^{-12}$ & 0.10 & 1800 & - & 25000 & $1.3 \times 10^{60}$ & 280 \\
& 160000 & $1.3 \times 10^{-12}$ & 0.086 & 4400 & - & $-{ }^{\prime \prime}-$ & $-"-_{-}^{\prime \prime}-$ \\
\hline
\end{tabular}

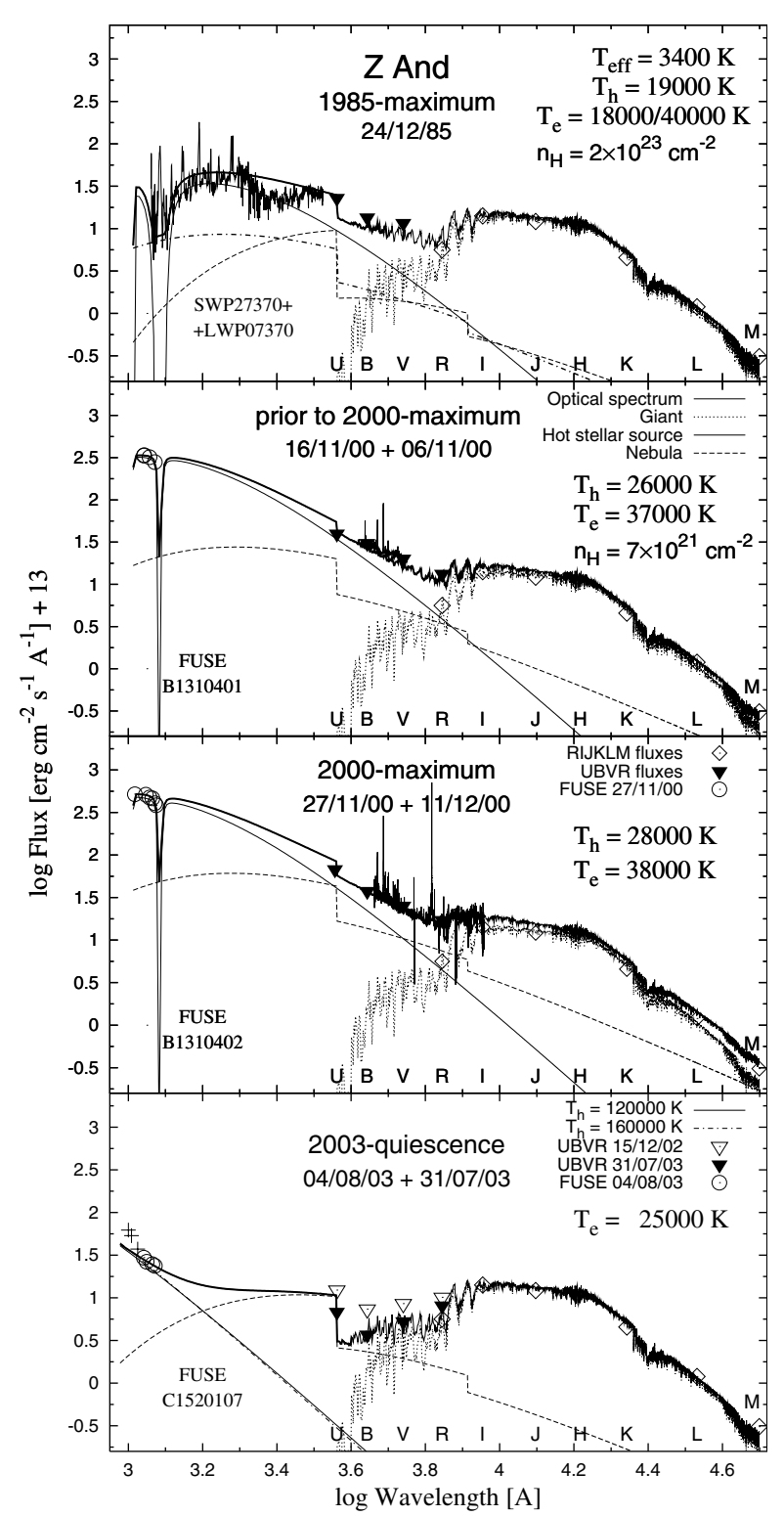

Fig. 7. The UV/optical/IR SED during active phases of Z And. Top: at the 1985-maximum. Middle two panels: prior to and at the maximum of the 2000 outburst (positions in the LC are shown in Fig. 1). Bottom: at the post-outburst stage in the summer of 2003. The resulting spectrum (solid thick lines) is given by superposition of nebular component(s) (dashed lines) and two stellar components, from the hot stellar source (solid thin lines) and the giant (dotted lines). IR fluxes $(\diamond)$ are from quiescence and far-UV fluxes $(\circ,+$, Sect. 2.2) are near-simultaneous to the optical observations.

particle bounded). Therefore the significant increase of the nebular emission during the maxima of the outbursts can result from a supplement of new emitters into the particle bounded nebula, accompanied probably by an increase in the flux of ionizing photons from the hot source. The former can be caused by an increase in the mass-loss rate from the hot object. The broad $\mathrm{H} \alpha$ wings (Fig. 4, Sect. 3.5) suggest the mass-outflow to be in the form of a stellar wind. Skopal et al. (2006) estimated the corresponding mass-loss rate at the maximum as $\sim 2 \times 10^{-6} M_{\odot} \mathrm{yr}^{-1}$, which is a factor of $\sim 3$ larger than that from the giant (see Table 3 in Skopal 2005). The presence of a rather high-temperature nebula $\left(T_{\mathrm{e}}=30000-40000 \mathrm{~K}\right.$, Table 2$)$ requires a large contribution from collisions (Gurzadyan 1997), which is consistent with the strong mass-outflow. Sokoloski et al. (2006) ascribed the increased X-ray emission at the optical maximum to the shockheated plasma as a consequence of the mass ejection from the white dwarf into the dense symbiotic nebula. The transient increase of the nebular emission during the low-amplitude 2002/03 rebrightening (bottom panel of Fig. 7) was also connected with a release of new emitters into the particle bounded nebula (see Sect. 3.6). Contrarily, during the plateau stages the SED did not change considerably as suggested by the stability of colour indices (Sect. 3.1). This constrains the hot object to be stable in the luminosity, temperature and also the mass outflow, because more emitters would give rise a surplus of the emission measure and thus the star's brightness.

\subsection{Direct and Raman scattered O vı 11032 line}

The Raman scattering process and its possible applications in astrophysics was introduced by Nussbaumer et al. (1989). In this process a photon excites an atom from its ground state to an intermediate state, which is immediately stabilized by a transition to a true bound state by emitting a photon of a different frequency. Schmid (1989b) identified the $\lambda 6830$ and $\lambda 7088$ emission bands observed in many symbiotic stars as to be due to Raman scattering of the O VI resonance doublet $\lambda 1032, \lambda 1038$ by neutral hydrogen. This process has been investigated by many authors (e.g. Schmid 1996; Harries \& Howarth 1996; Schmid et al. 1999; Birriel et al. 2000). The efficiency of this scattering process and profiles of both the direct and the Ramanscattered O VI lines together with polarimetric measurements provide information on the scattering geometry in symbiotic systems (Schmid 1998). Previous studies referred exclusively to quiescent phases of symbiotic stars. For Z And, Birriel et al. (1998) revealed strong evidence for the Raman effect and determined its efficiency to $\sim 7 \%$ for $\lambda 1032$ on the basis of the HUT observations. Schmid et al. (1999) derived the efficiency of this conversion to $4-6 \%$ and found that the Raman $\lambda 6825$ profile in $\mathrm{Z}$ And is by $30 \%$ broader than the original O VI $\lambda 1032$ line on the violet side of the profile. They used observations from the ORFEUS mission.

Here we analyze our series of Raman $\lambda 6830$ lines obtained along the major Z And outburst (Fig. 4) together with the archival FUSE spectra covering the same period. 
Table 3. Dereddened fluxes for the O VI $\lambda 1032$ line and its Raman scattered counterpart at $\lambda 6825$ in units of $10^{-12} \mathrm{erg} \mathrm{cm}^{-2} \mathrm{~s}^{-1}$. The efficiency, $\eta$, was derived according to the relation (3).

\begin{tabular}{cccccc}
\hline \hline Date & $\begin{array}{c}\text { Julian date } \\
24 \ldots\end{array}$ & Phase & $F_{\text {OvI }}$ & $F_{\text {Ram }}$ & $\begin{array}{c}\eta \\
{[\%]}\end{array}$ \\
\hline $16 / 11 / 00$ & 51865.0 & 0.16 & 50.6 & - & - \\
$27 / 11 / 00$ & 51876.0 & 0.18 & 44.6 & - & - \\
$10 / 12 / 00$ & 51889.4 & 0.19 & $45.4 \mathrm{i}$ & $<2^{\dagger}$ & $<29$ \\
$11 / 12 / 00$ & 51890.4 & 0.19 & $45.5 \mathrm{i}$ & $<2^{\dagger}$ & $<29$ \\
$12 / 12 / 00$ & 51891.3 & 0.20 & $45.6 \mathrm{i}$ & $<2^{\dagger}$ & $<29$ \\
$15 / 12 / 00$ & 51894.0 & 0.20 & 45.8 & - & - \\
$05 / 01 / 01$ & 51914.9 & 0.23 & $74.5 \mathrm{i}$ & $<2^{\dagger}$ & $<18$ \\
$27 / 05 / 01$ & 52057.3 & 0.42 & $270 . \mathrm{i}$ & $<2^{\dagger}$ & $<5$ \\
$22 / 07 / 01$ & 52113.3 & 0.49 & 347. & - & - \\
$30 / 09 / 01$ & 52183.3 & 0.58 & 308. & - & - \\
$10 / 02 / 02$ & 52315.9 & 0.76 & $972 . \mathrm{i}$ & 4.31 & 2.9 \\
$05 / 07 / 02$ & 52461.5 & 0.95 & 1700. & $8.93 \mathrm{i}$ & 3.5 \\
$07 / 08 / 02$ & 52494.3 & 0.99 & $1520 . \mathrm{i}$ & 9.97 & 4.3 \\
$22 / 10 / 02$ & 52570.5 & 0.09 & 1103. & $16.2 \mathrm{i}$ & 9.7 \\
$02 / 02 / 03$ & 52673.5 & 0.23 & $1303 . \mathrm{i}$ & 24.7 & 12.5 \\
$31 / 07 / 03$ & 52852.5 & 0.47 & $1650 . \mathrm{i}$ & 13.7 & 5.5 \\
$04 / 08 / 03$ & 52856.8 & 0.47 & 1658. & $13.8 \mathrm{i}$ & 5.5 \\
$02 / 09 / 03$ & 52885.5 & 0.51 & $1714 . \mathrm{e}$ & 14.4 & 5.6 \\
\hline
\end{tabular}

i/e: interpolated/extrapolated value,

$\dagger$ : no detection, upper limits only.

\subsubsection{Raman scattering efficiency}

The scattering efficiency for the Raman process is defined as the photon ratio, $\eta(\mathrm{OVI})=N_{\mathrm{Ram}} / N_{\mathrm{OVI}}$, between the Raman scattered and the initial O VI line component, i.e. the fraction of emitted O VI photons converted to Raman photons. As we measured only the Raman $\lambda 6825$ line we investigate here the conversion $\lambda 1032 \rightarrow \lambda 6825$. In the simplest case the efficiency of this transition can be written as

$\eta(\mathrm{O} \mathrm{VI})=6.614 \frac{F_{\mathrm{Ram}}}{F_{\mathrm{O} \text { vI }}^{\mathrm{obs}}}$

where the factor $6.614=\lambda 1031.92 / \lambda 6825.44$. In the real case $\eta$ depends on the geometry of H I atoms within the binary and the binary position with respect to the observer (Schmid 1996). Thus the relation (3) represents an empirical measure of the line photon ratio we call efficiency. Table 3 and Fig. 8 summarize the results. As the FUSE and our optical observations were not simultaneous, we interpolated FUSE fluxes to dates of optical observations and vice versa. Below we comment on the variation in $\eta$ along the outburst as follows:

(i) At the initial stages (2000-01) the unmeasurable Raman band could be a consequence of a very low O VI $\lambda 1032$ flux. Assuming the conversion efficiency of a few percent, the incident flux of $45 \times 10^{-12} \mathrm{erg} \mathrm{cm}^{-2} \mathrm{~s}^{-1}$ (Table 3) produces a few times $10^{-13} \mathrm{erg} \mathrm{cm}^{-2} \mathrm{~s}^{-1}$ at $\lambda 6825$, which implies the height of the Raman feature above the local continuum of only a few times $10^{-14} \mathrm{erg} \mathrm{cm}^{-2} \mathrm{~s}^{-1}$, which is under the level of detection. On the other hand, the signal to noise ratio $S / N \sim$ 20 around the Raman line suggests a limiting flux of $\sim 1 \times$ $10^{-13} \mathrm{erg} \mathrm{cm}^{-2} \mathrm{~s}^{-1} \AA^{-1}$ needed to recognize a detail in the spectrum. Then, assuming the Raman line to be flat and as broad as $\sim 20 \AA$, its flux undetectable by us is $\lesssim 2 \times 10^{-12} \mathrm{erg} \mathrm{cm}^{-2} \mathrm{~s}^{-1}$. The corresponding upper limits for efficiencies $\eta$ are relatively high, because of low initial O VI fluxes at the optical maximum (Table 3, Eq. (3), Appendix B).

(ii) In 2002, prior to the spectroscopic conjunction, the emergence of the Raman features and the enhancement of the

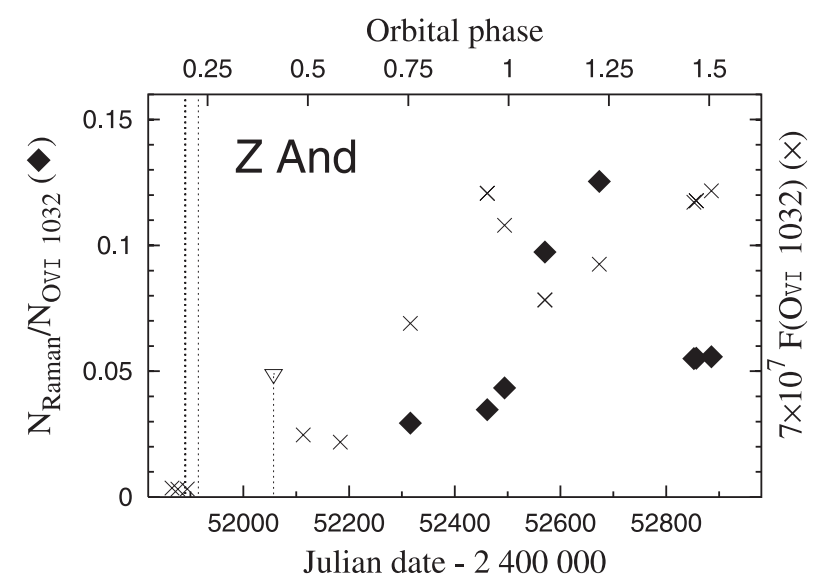

Fig. 8. Evolution of the Raman conversion efficiency $\lambda 1032 \rightarrow \lambda 6825$ along the outburst of $\mathrm{Z}$ And (full diamonds). Open triangles connected with a dotted line denote upper limits for Raman fluxes that were below our detection level (see the text). Data are from Table 3.

O VI fluxes (Table 3, Fig. 8) require extension of the scattering region to obtain the measured efficiencies of $3-4 \%$. Note that the flux increase of the $\lambda 1032$ line itself would result in a decrease of the conversion efficiency (Eq. (3)). A comparison of the derived efficiencies to a grid of model calculations published by Schmid (1996) suggests that his XB3 model could be relevant, i.e. nearly one third of the sky, as seen from the O VI ionized zone, is covered by the neutral scattering region.

(iii) A significant increase in the Raman conversion efficiency during the first quadrature after the spectroscopic conjunction (2002 October-2003 March, orbital phases 0-0.25, Fig. 8) reflects an enlargement of the neutral region seen from the O VI zone. The observed O VI $\lambda 1032$ flux decreased, while its Raman scattered counterpart at $\lambda 6825$ increased (Table 3 ). Here the efficiency of $10-12 \%$ is similar to that of the $X C 3$ model of Schmid's (1996) simulations, in which at least one half of the "O VI sky" is occupied by atoms of neutral hydrogen. This change in distribution of the neutral material in the binary could be connected with an enhancement of the hot star wind near the equatorial plane as a result of dilution of the optically thick disklike shell during this period (Sect. 4). According to hydrodynamical calculations that include effects of the orbital motion, a dense S-shaped wind-wind collision region can be formed between the stars where the fast wind pushes the giant wind (e.g. Fig. 3 of Walder 1995). This interaction zone can divide the binary space into two approximately equal parts of the neutral and ionized wind. Such a distribution of a neutral giant's wind in an active binary satisfies conditions required by the higher efficiency of the Raman scattering process.

(vi) During the post-outburst period (2003, orbital phase $\sim 0.5$ ), observed OVI fluxes increased, while the $\lambda 1032 \rightarrow \lambda 6825$ conversion efficiency decreased. This indicates a decrease in the size of the scattering region as "seen" by the O VI photons. Derived efficiencies of $\sim 5 \%$ are similar to those in quiescence (Birriel et al. 1998; Schmid et al. 1999).

\subsubsection{Comparison of line profiles}

Evolution in the Raman-scattered O VI $\lambda 1032$ line along the outburst is shown in Fig. 4. A comparison of both direct and Raman-scattered lines is plotted in Fig. 9 for near-simultaneous optical/far-UV observations. Table 4 summarizes their basic parameters $\left(R V_{\mathrm{c}}, F W H M\right)$. A well-measurable Raman $\lambda 6825$ line 
developed on $07 / 08 / 02$ on our spectra, i.e. nearly two years after the beginning of the outburst. Profiles consist of a central peak at $\sim 6832 \AA$ and a violet and red shoulder at $\sim 6823 \AA$ and $\sim 6837 \AA$, respectively. On $02 / 02 / 03$ the red adjacent component developed in a separate peak of a comparable intensity to the main peak. The directly measured O VI $\lambda 1032$ line was characterized by a strong central emission shifted by about $+58 \mathrm{~km} \mathrm{~s}^{-1}$ with respect to the systemic velocity. Such a large redshift was measured also during a quiescent phase by Schmid et al. (1999) who explained this effect by radiative transfer in strong resonance lines throughout an expanding medium. During the active phase, this line absorption/scattering effect attenuated the violet wing from about +50 to $-350 \mathrm{~km} \mathrm{~s}^{-1}$ and produced a P-Cygni type of profile (Fig. 3). This suggests its origin in the densest part of the hot star wind. Other variations along the active phase (Fig. 9) are discussed below.

We compare the line profiles in the radial velocity (RV) space considering the principle of energy conservation of the Raman scattering process. According to Nussbaumer et al. (1989) a broadening $\Delta \lambda_{\mathrm{i}}$ of the incident line $\lambda_{\mathrm{i}}$ is transferred to a broadening $\Delta \lambda_{\mathrm{f}}$ of the scattered line $\lambda_{\mathrm{f}}$ as

$\frac{\Delta \lambda_{\mathrm{f}}}{\lambda_{\mathrm{f}}}=\frac{\Delta \lambda_{\mathrm{i}}}{\lambda_{\mathrm{i}}} \frac{\lambda_{\mathrm{f}}}{\lambda_{\mathrm{i}}}$

In the RV-scale and for the O VI $(\lambda 1032 \rightarrow \lambda 6825)$ scattering process, Eq. (4) can be written as $\Delta R V_{\mathrm{OVI}}=\Delta R V_{\text {Ram }} / 6.614$. Further we assume that: (a) the OVI emission region is connected with the wind from the hot star. (b) The scattering region is represented by neutral atoms of hydrogen from the wind of the giant. (c) Between the binary components there is an interaction zone of the two winds. It separates a low density (filled with material from the hot star) and a high density region (filled with material from the giant). The zone can be spiral-shaped due the orbital motion (e.g. Walder 1995). From a general discussion on the O VI line profiles of 6 objects such as given by Schmid et al. (1999), we propose the following explanation of our observations (Fig. 9):

(i) The first measured Raman $\lambda 6825$ line $(10 / 02 / 02, \varphi=$ 0.76) was compared with the direct O VI line from 30/09/01 $(\varphi=0.58)$. Unusually, with respect to later observations, both profiles nearly overlapped. Thus there was no significant motion of the scattering region relative to the direction of the incoming O VI photons. This implies that the O VI photons could not impact the neutral wind on the orbital plane moving toward them. Otherwise the $\sim-40 \mathrm{~km} \mathrm{~s}^{-1}$ shift of the Raman line relative to the original line should have been observed (Schmid et al. 1999). According to our interpretation of the hot object structure (Sect. 4), disk-like material blocked the O VI photons at the orbital plane, which thus could "see" only outer parts of the giant's wind giving rise to only a small redshift in the $\lambda 6825$ profile. Note that the major part of the O VI flux is created in the densest regions of the wind encompassing the hot star (cf. Appendix B). Another peculiarity is that the violet wing of the $\lambda 1032$ line was not so strongly absorbed in the wind medium as we observed later during the outburst. As a result the total profile was shifted only to $\sim+35 \mathrm{~km} \mathrm{~s}^{-1}$ (Table 4). A shallow minimum in the $\lambda 1032$ line at $\sim+18 \mathrm{~km} \mathrm{~s}^{-1}$ could be caused by a self-absorption near to the hot object. Note that the radial velocity of the hot component orbital motion at the time of the observation $(\varphi=0.76)$ is comparable to this shift (parameters from Mikolajewska \& Kenyon 1996), which thus places its origin at/around the hot star, in agreement with our assumption (a) above.

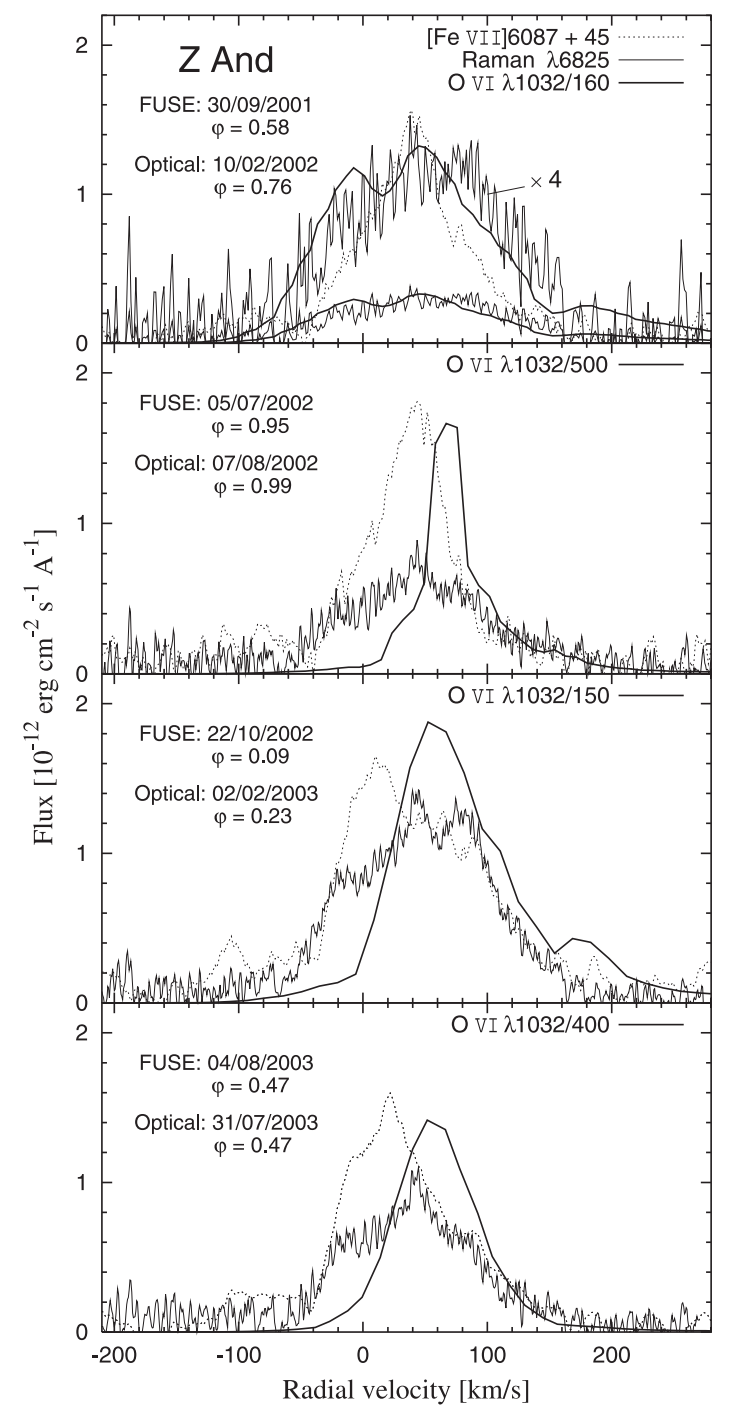

Fig. 9. Comparison of the OVI $\lambda 1032$ line profiles with their Raman scattered counterparts during the outburst. The [Fe VII] $\lambda 6087$ line profile shifted by $+45 \mathrm{~km} \mathrm{~s}^{-1}$ (see Sect. 3.4.3) is compared. The O VI line flux was scaled to that of the Raman line.

(ii) The next available pair of O VI $\lambda 1032$ and $\lambda 6825$ lines was taken just prior to the inferior conjunction of the red giant $(\varphi=0.95$ and 0.99$)$. The line flux at $\lambda 1032$ was the largest from our sample and its profile was characterized by a narrow redshifted core $\left(R V_{\mathrm{c}} \sim+67 \mathrm{~km} \mathrm{~s}^{-1}\right)$, while its scattered counterpart at $\lambda 6825$ was rather faint. The profile of the initial O VI line was probably modified by an occultation of the O VI nebula, which thus reduced its violet wing for the outer observer. Note that from $\varphi \sim 0.94$ a decrease of the optical light due to the eclipse effect was observed (Fig. 2 of Skopal 2003a). On the other hand, the O VI zone was not subject to occultation for the scattering region spread within the H I zone in the binary. Thus, this could "see" the un-affected O VI $\lambda 1032$ line profile and convert it to a standard type of the $\lambda 6825$ line profile as measured at other orbital phases later on. The significant increase of the direct O VI line flux at approximately unchanged or slightly increased conversion efficiency constrains the enhancement of the $\mathrm{H}$ I atoms taking part in the scattering process (Eq. (3)). The former resulted from the temperature increase of the ionizing source (Sects. 3.2 (vii) and 3.3), while the latter could result from a dilution of the cooler disk-like pseudophotosphere. As 
Table 4. Line center $\left(R V_{\mathrm{c}}\right)$ and line width $(F W H M)$ for direct and Raman scattered O VI $\lambda 1032$ lines in $\mathrm{km} \mathrm{s}^{-1}$.

\begin{tabular}{|c|c|c|c|c|}
\hline \multirow[t]{2}{*}{$\overline{\overline{\text { Date }}}$} & \multicolumn{2}{|c|}{$R V_{\mathrm{c}}$} & \multicolumn{2}{|c|}{$\overline{F W H M}$} \\
\hline & $\lambda 6825$ & $\lambda 1032$ & $\lambda 6825$ & $\lambda 1032$ \\
\hline $22 / 07 / 01$ & - & 30 & - & 145 \\
\hline $30 / 09 / 01$ & - & 35 & - & 150 \\
\hline $10 / 02 / 02$ & 46 & - & 160 & - \\
\hline 05/07/02 & - & 67 & - & 34 \\
\hline 07/08/02 & 36 & - & 120 & - \\
\hline $22 / 10 / 02$ & - & 64 & - & 93 \\
\hline $02 / 02 / 03$ & 41 & - & 139 & - \\
\hline $31 / 07 / 03$ & 33 & - & 118 & - \\
\hline $04 / 08 / 03$ & - & 59 & - & 77 \\
\hline 02/09/03 & 35 & - & 120 & - \\
\hline \multicolumn{5}{|c|}{ Quiescent phase } \\
\hline $31 / 01 / 95^{\dagger}$ & - & - & $\approx 113$ & - \\
\hline $06 / 03 / 95^{\dagger}$ & - & - & - & 70 \\
\hline $28 / 11 / 96^{\ddagger}$ & 33 & 57 & 101 & 78 \\
\hline
\end{tabular}

a result the wind from the hot star could appear close to the orbital plane and thus met a massive neutral wind from the giant moving against it. This lead to the appearance of the violet adjacent component in the $\lambda 6830$ profile and an enhancement of the $\eta(\mathrm{O}$ VI) efficiency.

(iii) After the spectroscopic conjunction (the far-UV and optical observation made at $\varphi=0.09$ and 0.23 , respectively; Table 3), during the 2002/03 transient increase in the star's brightness, we observed the largest efficiency of the $\lambda 1032 \rightarrow$ $\lambda 6825$ transition, a well developed three-peaked structure of the Raman line and a relatively broad line core of the direct $\mathrm{O}$ VI line with superposition of a wind component at $\sim+180 \mathrm{~km} \mathrm{~s}^{-1}$ (Fig. 9). The Raman line was made broader by the blue adjacent component. Its wing was shifted by about $-40 \mathrm{~km} \mathrm{~s}^{-1}$ relative to that of the $\lambda 1032$ line. This emission is produced by the HI atoms of the giant's wind, which are located along the binary axis and move toward the O VI region. The absorption component in the emission core of the $\mathrm{H} \alpha$ profile at the position of $\approx-45 \mathrm{~km} \mathrm{~s}^{-1}$ (Sect. 3.2 (ii)) supports this interpretation. In addition, a dense neutral region could be created as an enhanced hot star wind pushes the neutral giant's wind between the binary components. This can increase the effective number of hydrogen atoms and thus also the efficiency $\eta(\mathrm{O} \mathrm{VI})$. The outer part of the neutral wind and the $\mathrm{HI}$ atoms in the collisional zone moving from the incoming O VI photons then extend the red side of the Raman line profile. The red wind component in the $\lambda 1032$ profile has no Raman counterpart. This portion of the O VI emission has an origin at the outer part of the hot wind, i.e. further away from the scattering region, which reduces the scattering efficiency.

(iv) Similar profiles were observed later at the orbital phase $\varphi=0.5$ (nearly simultaneous observations, bottom panel of Fig. 9). This suggests the difference between the direct and the Raman line profile to be of the same nature, as we discussed above in point 3 .

The shift between the red wings of the Raman-scattered and the direct $\mathrm{O}$ VI line during quiescent phase was larger than we observed during activity. The FWHM of the Raman line in activity exceeded this quantity in quiescence by about $10-30 \mathrm{~km} \mathrm{~s}^{-1}$, while the width of the incident OVI line did not change much (Schmid et al. 1999; Birriel et al. 2000, Table 4 here). This reflects different kinematics of the scattering region during active and quiescent phases. Because of a strong wind from the hot star developed during the active phase, this could be due to the wind-wind interaction zone in the binary. Therefore, in the following section, we discuss possible connections between profiles of the [Fe VII] $\lambda 6087$ and the Raman $\lambda 6825$ line.

\subsubsection{Raman $\lambda 6825$ and [Fe VII] $\lambda 6087$ profiles}

A comparison of [Fe VII] $\lambda 6087$ and Raman $\lambda 6825$ line profiles is shown in Fig. 9. The former was shifted by $+45 \mathrm{~km} \mathrm{~s}^{-1}$ to eliminate the redshift in the Raman line (Sect. 3.4.2). The similarity of both profiles is clear in 2002 and 2003 when also their line fluxes were comparable. Their profiles - a large FWHM of $\approx 120-140 \mathrm{~km} \mathrm{~s}^{-1}$, steep wings with a multi-peak structure at the top - suggest that they are due to the kinematics of emitting regions. The wings of both lines overlap at least up to half of the maximum. The nearly equality of the extended red wings with a pattern of the red adjacent component is striking (bottom panels of Fig. 9).

These common signatures of both line profiles suggest similar kinematics for the responsible emitting regions, but that differs drastically in conditions of ionization - one region has to contain $\mathrm{HI}$ atoms at high density, while the other has $\mathrm{Fe}^{+6}$ ions at low density. This can be satisfied if these lines are formed within the wind-wind interaction zone. The Raman line can be created at/around the interaction surface on the giant's side, where its neutral wind is pushed by the hot star wind, while the [Fe VII] emission can arise in a collisionally ionized boundary layer between the two winds. The observed change in the line width at $\varphi \sim 0.25(02 / 02 / 03$, a maximum width) and $\varphi \sim 0.5$ $(31 / 07 / 03$, a narrower width) is consistent with the main wind flow velocity aligned approximately perpendicular to the line connecting the stars. Previous works also support such an origin of [Fe VII] lines. Wallerstein et al. (1984) showed that line profiles of forbidden lines from highly ionized atoms could result from interaction nebula of wind-wind collision in a binary system. The observed profile of the [Fe VII] line in V1016 Cyg was very similar to our observations from 2003 (see their Fig. 4). Hydrodynamical calculations of the colliding zone of the two winds by Nussbaumer \& Walder (1993) showed a wide variety of resulting line profiles, some of which are similar to our observations (e.g. the overall broadening with steep sides of profiles).

Accordingly the observed broadening of the Raman line profile together with profiles of highly ionized forbidden lines can provide a diagnostic tool in studying motion in the wind-wind interaction zone during active phases. Note that a Raman photon may escape easily from the neutral region, because this elastic process cannot be repeated (in contrast to Rayleigh scattering) and thus reflects motion in the densest (a small cross-section) outer layers illuminated by $\mathrm{O}$ VI photons.

\subsection{On the origin of the broad $\mathrm{H} \alpha_{\alpha}$ wings}

Nussbaumer et al. (1989) pointed out that the broad $\mathrm{H} \alpha$ wings in symbiotic stars could be formed through Raman scattering of Ly $\beta$ photons by atomic hydrogen. Lee (2000) and Lee \& Hyung (2000) elaborated a relevant model. They showed that the wing profile formed through Raman scattering can be approximated by the curve proportional to $f(\Delta v) \propto \Delta v^{-2}$ in the velocity coordinates. On the other hand, Skopal et al. (2002a) derived an expression of the same type (see their Eq. (11)) to fit the broad components of the hydrogen line profiles that form in an optically thin and spherically symmetric stellar wind. Therefore it is not possible to distinguish the wind emission from the scattered features in the $\mathrm{H} \alpha$ wing directly. In addition, the observed 
Ly $\beta$ profile can be influenced by other processes in the circumstellar medium.

In our case the observed $\mathrm{Ly} \beta$ profile is not able to produce the $\mathrm{H} \alpha$ wings through the Raman scattering process. The width of the scattered $\mathrm{H} \alpha$ wings is proportional to the width of the $\operatorname{Ly} \beta$ line as $\Delta v_{\mathrm{Ly} \beta}=\Delta v_{\mathrm{H} \alpha} / 6.4$. Then the full extension of $\mathrm{H} \alpha$ wings $\gtrsim 4000 \mathrm{~km} \mathrm{~s}^{-1}$ (Fig. 4) requires $\Delta v_{\mathrm{Ly} \beta}>625 \mathrm{~km} \mathrm{~s}^{-1}$ or $\Delta \lambda_{\mathrm{Ly} \beta}>2.1 \AA$, which is a factor of $\sim 2$ larger than the observed $F W Z I(\operatorname{Ly} \beta)<1.0 \AA$. In the case of an optically thick Rayleigh scattering region the $\mathrm{Ly} \beta$ photons may undergo many Rayleigh scatterings until they are converted into Raman photons and escape. Thus in such a special case only Raman features can be detected, but no original Ly $\beta$ emission. The real case is complicated by the presence of a strong and variable geocoronal component of $\operatorname{Ly} \beta$, which is difficult to isolate from the circumstellar one. As a result we have to investigate other possibilities to identify the main source of radiation contributing to the broad $\mathrm{H} \alpha$ wings. For example, Ikeda et al. (2004) found that the polarization profile of $\mathrm{H} \alpha$ does not agree with that of the Raman $\lambda 6830$ line for $Z$ And on 25/10/02 (i.e. during the 2002/03 brightening). This finding suggests that the $\mathrm{H} \alpha$ line does not include the Raman-scattered component that originates from Ly $\beta$. Also a relationship between the emission from $\mathrm{H} \alpha$ wings and the emission measure of the symbiotic nebula supports the origin of the broad $\mathrm{H} \alpha$ wings in the ionized stellar wind (Skopal 2006).

Therefore we suggest that the extended $\mathrm{H} \alpha$ wings, which developed during the investigated active phase of $\mathrm{Z}$ And, are due to kinematics of the ionized hydrogen rather than the Raman scattered $\operatorname{Ly} \beta$ photons. However, this problem deserves further investigation.

\subsection{Optical rebrightening in $2002 / 03$}

After 2002 September/October, when the hot object was rising from the eclipse, a rebrightening in the LC $(\Delta U \sim 0.6$, $\Delta B \sim 0.4, \Delta V \sim 0.3$ ) was observed for about 5 months. Our estimate of its magnitude increase takes into account the depth of the eclipse and the brightness level prior to it (Skopal 2003a). During this event we measured an additional extension of the $\mathrm{H} \alpha$ wings on our spectrum from 02/02/03 and Tomov et al. (2005) observed such broadening also in the emission wings of the He II $\lambda 4686$ line profile with $F W Z I \sim 2400 \mathrm{~km} \mathrm{~s}^{-1}$.

The observed indices from the maximum (around $15 / 12 / 2002)$ were $(U-B)_{\mathrm{obs}}^{\max } \doteq-0.70$ and $(B-V)_{\mathrm{obs}}^{\max } \doteq 0.85$. If we deredden these observations with $E(B-V)=0.30$, correct for emission lines (we adopted $\Delta U_{l}=0.25, \Delta B_{l}=0.45$ and $\Delta V_{l}=0.18$ corresponding to a standard emission line spectrum for classical symbiotics (Skopal 2003b)) and subtract contributions from the giant, $F_{\mathrm{g}}(B)=1.3 \times 10^{-13} \mathrm{erg} \mathrm{cm}^{-2} \mathrm{~s}^{-1} \AA^{-1}$ and $F_{\mathrm{g}}(V)=2.9 \times 10^{-13} \mathrm{erg} \mathrm{cm}^{-2} \mathrm{~s}^{-1} \AA^{-1}$ (see Fig. 7), we get indices of the extra light that causes the optical rebrightening as $(U-B)_{\text {extra }}^{\max } \doteq-1.27$ and $(B-V)_{\text {extra }}^{\max } \doteq 0.59$. These quantities reflect a large contribution from the nebula radiating at high electron temperature (e.g. Menzies et al. 1982). We demonstrate this case in Fig. 7. The $U B V R$ flux-points from the maximum of the 2002/03 rebrightening suggest the same type of the SED as in the mid-2003, but with a factor of $\sim 2$ stronger contribution from the nebula $\left(E M(\right.$ rebrightening $\left.) \approx 2.6 \times 10^{60} \mathrm{~cm}^{-3}\right)$.

Assuming a particle-bounded nebula (i.e. geometrically open in the sense of the STB model), a maximum increase of its radiation at a constant flux of ionizing photons can be produced by an injection of new particles into it, that will convert a relevant amount of the ionizing photons into the nebular radiation to yield a radiation-bounded (closed) nebula. The particle-bounded nebula in Z And during 2002-03 is supported by a high quantity of the hydrogen ionizing photons of $z 1.2 \times 10^{47} \mathrm{~s}^{-1}$ given by the hot object luminosity $\left(L_{\mathrm{h}}>1800 L_{\odot}\right)$ and its temperature $\left(T_{\mathrm{h}}>120000 \mathrm{~K}\right.$, Table 2$)$. A large value of the parameter $X(\approx 14)$ during quiescence corresponding to an open nebula was noted by Fernández-Castro et al. (1988). Within this scenario we interpret the small optical rebrightening as a consequence of a dilution of the optically thick shell around the hot star. This event supplied additional emitters into the particlebounded nebula, which can convert a relevant part of the farUV radiation through $f-b$ and $f-f$ transitions to the optical. Thus, the material became more transparent and thus was subject to ionization by the hot central star even on the orbital plane, which led to a transient increase of the nebular emission. A similar brightening was also observed in the LC of AX Per at its transition from the active to quiescent phase (Figs. 3 and 10 of Skopal et al. 2001). Dilution of an optically thick shell in this case was directly confirmed by the change of the narrow eclipses into very broad minima occurring just after the event (cf. Fig. 3 there).

Sokoloski et al. (2006) interpreted the 2002/03 rebrightening as a result of a decrease in $T_{\mathrm{h}}$ (from around $150000 \mathrm{~K}$ to approximately $120000 \mathrm{~K}$ ) due to a slight expansion of the whitedwarf photosphere. They based their explanation on that both the FUSE and VLA fluxes were unusually low during the optical rebrightening and the $U-B$ index varied from about -0.45 to -0.8 from the beginning to the end of the event. We suggest the following arguments against this interpretation: (i) the decrease in the $F U V$ fluxes and $T_{\mathrm{h}}$ implies a decrease in both the stellar and the nebular component of radiation from the hot object. Such the small temperature decrease at $T_{\mathrm{h}}>100000 \mathrm{~K}$ induces a negligible (i.e. not measurable) change in the SED (e.g. Munari \& Buson 1992). Thus, we should observe a decrease of the optical brightness. (ii) There is an additional attenuation of the UV continuum that is clearly seen in eclipsing systems at positions around the inferior conjunction of the giant. This extinction process was first noticed by Mürset et al. (1991) and Dumm et al. (1999) analyzed it for RW Hya. Skopal (2005) found this effect to be present in all IUE spectra of Z And in the range of phases $\varphi \approx 0 \pm 0.15$. Therefore the "unusually" low FUSE fluxes from 22/10/2002 $(\varphi=0.093)$ result from this effect. (iii) The observed fading in the radio fluxes is consistent with that in $E M: E M($ maximum $) / E M(2002 / 03) \approx$ $F_{5 \mathrm{GHz}}($ maximum $) / F_{5 \mathrm{GHz}}(2002 / 03)$ within the corresponding uncertainties (Table 2 here and Table 5 of Sokoloski et al. 2006). (iv) A redder $U-B$ index at the beginning of the rebrightening $(-0.45)$ and a more blue value at/after the maximum $(-0.8)$ could qualitatively reflect variation in optical properties of the ionized medium during/after the shell dilution.

\section{Structure of the hot object during the outburst}

\subsection{The stage at/around the optical maximum}

This stage was characterized by:

(i) A two-temperature-type hot object spectrum. The cooler spectrum was produced by a relatively warm stellar source radiating at 20000-30000 $\mathrm{K}$ (Fig. 7, Table 2) and the hotter one was represented by the highly ionized emission lines (e.g. He II, O VI, [Fe VII]) and a strong nebular continuum radiation ( $E M \gtrsim 10^{60} \mathrm{~cm}^{-3}$, Table 2). The former is not capable of producing the observed nebular emission and thus the latter signals the presence of a hot ionizing source $\left(\gtrsim 10^{5} \mathrm{~K}\right)$ 
in the system, which is not seen directly by the outer observer.

(ii) Signatures of a mass outflow at moderate velocities $\left(\sim 100-200 \mathrm{~km} \mathrm{~s}^{-1}\right)$, as indicated by the P-Cygni profiles (Sect. 3.2 (i), Figs. 2 and 6; also Sokoloski et al. 2006), and at very high velocities $\left(\approx 1000-2000 \mathrm{~km} \mathrm{~s}^{-1}\right)$ in broad emission wings of $\mathrm{H} \alpha$ (Fig. 4).

The two-temperature spectrum and the two-velocity type of the mass outflow from the hot object suggest an optically thick disklike structured material surrounding the central hot star expanding at moderate velocities in the orbital plane, while at higher latitudes a fast optically thin wind escapes the star. Due to a high orbital inclination the outer observer can see just the optically thick matter of the disk-like shaped warm pseudophotosphere, whose outer flared rim occults the central ionizing source. Only the surrounding medium from above/below the disk can directly interact with the ionizing source and thus convert its radiation to nebular emission. As a result, the temperature of the ionizing source, $T_{\mathrm{h}}^{\text {i.s. }}$, can be obtained only through emission lines (Sokoloski et al. 2006, derived $T_{\mathrm{h}}^{\text {i.s. }} \sim 95000 \mathrm{~K}$ ), while the observed UV/optical continuum determines the temperature of the stellar source (i.e. the warm pseudophotosphere), $T_{\mathrm{h}} \sim 27000 \mathrm{~K}$ (Sect. 3.3). Thus this stage is characterized by

$T_{\mathrm{h}} \ll T_{\mathrm{h}}^{\mathrm{i} . \mathrm{s}}$.

The low fluxes of highly ionized elements, whose profiles were shifted blueward (Sect. 3.2 (iii), Fig. 2 for He II, Fig. 4 for [Fe VII]), are consistent with a disk-like structure of the hot object. The small size of the corresponding ionized zone (see Appendix B) produced relatively weak emission and the optically thick disk-like extended source, inclined at $75-80^{\circ}$ (Skopal 2003a), blocked a fraction of the radiation from outflow at its back side in the direction of the observer. As a result the blueward shifted emission dominated the profile. This basic structure of the active object persisted probably until about 2002 August, when the eclipse effect in the LC was the last indication of the presence of the optically thick disk-like shell around the hot star (Skopal 2003a).

This structure of the hot object is common for all active S-type symbiotics with a high orbital inclination (Skopal 2005, Fig. 27).

\subsection{Evolution after 2002 August}

During this period we see a more significant dilution of the optically thick material around the accretor and evolution of a fast wind from the hot star near the orbital plane. The latter is a direct consequence of the former event, because the flux of the hot radiation and particles from the accretor was less blocked in the direction of the orbital plane. These events are supported by the following results:

(i) A significant increase in the temperature of the stellar component of radiation, $T_{\mathrm{h}}$, we indicated through the model SED (Sect. 3.3.3) and by stronger fluxes of highly ionized elements (e.g. Fig. 5). Consequently, we have

$T_{\mathrm{h}} \sim T_{\mathrm{h}}^{\text {i.s. }}$,

in contrast to the evolution at/around the maximum (Eq. (5)).

(ii) The optical rebrightening during 2002.7-2003.3, which led to an enhancement of the nebular emission (Sect. 3.6). (iii) An additional extension of the $\mathrm{H} \alpha$ wings in our spectrum from $02 / 02 / 03$. Its temporarily larger terminal velocity and flux (Fig. 4) are consistent with the evolution of the hot star wind near the orbital plane and the enhancement of $E M$ (Sect. 3.6). Similar broadening was also measured for the He II $\lambda 4686$ line by Tomov et al. (2005).

(iv) The appearance of a fast wind from the hot star near the equatorial plane was also suggested by the [Fe VII] $\lambda 6087$ line profile, which evolved to that arising from the windwind interaction zone (Sect. 3.4.3).

(v) A sudden increase of the Raman conversion efficiency (Sect. 3.4.1, Fig. 8) and the appearance of the pronounced violet-shifted component in the Raman line profile (Sect. 3.4.2, Fig. 9). Both events need an increase in scattering; the latter also requires negative velocities between the $\mathrm{HI}$ atoms and $\mathrm{O}$ VI photons. This can be satisfied if a fast wind from the accretor and ionizing photons appear near the orbital plane and thus interact with the densest portion of the neutral wind from the giant around the binary axis that moves against the $\mathrm{O}$ VI photons.

Finally, the model SED from 2003 suggests that we still could not see the bare white dwarf, because of a large effective hot stellar radius $\left(R_{\mathrm{h}}^{\mathrm{eff}} \sim 0.1 R_{\odot}\right.$, Table 2$)$.

\section{Conclusions}

We studied the symbiotic prototype Z And using far-UV, optical low- and high-resolution spectroscopy and $U B V R$ broad-band photometry carried out during its major 2000-03 outburst. We summarize the main results as follows:

(i) An expansion of the hot object and an enhanced mass loss from the system was indicated by the change in the SED during the transition from the second plateau stage to the maximum (Sect. 3.3.2). A complex mass outflow from the system was recognized in the structure of the [O III] $\lambda 5007$ profiles (ejection of blobs, creation of adjacent emission components; Sect. 3.2, Fig. 2) and of the absorption components in C III and P V lines in the FUSE spectra.

(ii) Evolution of the two-temperature type of the spectrum at the initial stages of the outburst having signatures of a mass-outflow at moderate and high velocities suggested that the active object consists of optically thick, slowly expanding disk-like structured material encompassing the white dwarf at the orbital plane and of a fast optically thin wind escaping the star at higher latitudes (Sect. 4.1). The optically thick disk-like shell probably persisted until 2002 August. Afterwards we observed its dilution and evolution of a fast hot star wind concentrated more at the orbital plane (Sect. 4.2).

(iii) A striking similarity between [Fe VII] $\lambda 6087$ and Raman $\lambda 6825$ profiles at/after the dilution of the disk suggests their origin within the interaction zone, where the winds from both stars collide (Sect. 3.4.3). This unusual symbiosis of these lines and properties of the Raman scattering process can help to study streaming along the zone of interaction between the two winds.

Acknowledgements. The authors thank the anonymous referee for several helpful comments. Hans Martin Schmid is thanked for useful discussion about the Raman scattering process. M.W. thanks M. Šlechta for initial reduction of the spectra taken at the Ondřejov Observatory. This research was supported in part by a NATO Science Programme within its sub-programme EXPERT VISIT (A.S.), by a grant of the Slovak Academy of Sciences No. 2/4014/04 and the Grant Agency of the Czech Republic, grant No. 205/04/2063. The far ultraviolet data 
presented in this paper were obtained from the Multimission Archive at the Space Telescope Science Institute (MAST). They were made with the NASA-CNESCSA Far Ultraviolet Spectroscopic Explorer. FUSE is operated for NASA by the Johns Hopkins University under NASA contract NAS5-32985. A.S. acknowledges the hospitality of the Capodimonte Astrophysical Observatory in Naples.

\section{References}

Asplund, M., Grevesse, N., Sauval, A. J., et al. 2004, A\&A, 417, 751

Belyakina, T. S. 1985, IBVS, 2698

Belyakina, T. S. 1992, Izv. Krym. Astrofiz. Obs., 84, 49

Birriel, J. J., Espey, B. R., \& Schulte-Ladbeck, R. E. 1998, ApJ, 507, L75

Birriel, J. J., Espey, B. R., \& Schulte-Ladbeck, R. E. 2000, ApJ, 545, 1020

Bessell, M. S. 1983, PASP, 95, 480

Brocksopp, C., Sokoloski, J. L., Kaiser, C., et al. 2004, MNRAS, 347, 430

Castor, J. I., Abbott, D. C., \& Klein R. I. 1975, ApJ, 195, 157

Cardelli, J. A., Clayton, G. C., \& Mathis, J. S. 1989, ApJ, 345, 245

Chochol, D., \& Pribulla, T. 1997, Contrib. Astron. Obs. Skalnaté Pleso, 27, 53

Chochol, D., Grygar, J., Pribulla, T., et al. 1997, A\&A, 318, 908

Clegg, R. E. S. 1987, MNRAS, 229, 31

Crowley, C., Espey, B. R., \& McCandliss, S. R. 2004, in 13th Cool Stars Workshop, ed. F. Fawata et al., 1 [arXiv: astro-ph/0409417]

Dumm, T., Schmutz, W., Schild, H., \& Nussbaumer, H. 1999, A\&A, 349, 169

Fernández-Castro, T., Cassatella, A., Giménez, A., \& Viotti, R. 1988, ApJ, 324, 1016

Fernández-Castro, T., González-Riestra, R., Cassatella, A., et al. 1995, ApJ, 442, 366

Girard, T., \& Willson, L. A. 1987, A\&A, 183, 247

Gurzadyan, G. A. 1997, The Physics and Dynamics of Planetary Nebulae (Berlin: Springer-Verlag), 50

Harries, T. J., \& Howarth, I. D. 1996, A\&AS, 119, 61

Hauschildt, P. H., Allard, F., Ferguson, J., et al. 1999, ApJ, 525, 871

Henden, A. A., \& Kaitchuck, R. H. 1982, Astronomical Photometry (New York: Van Nostrand Reinhold Company), 50

Ikeda, Y., Akitaya, H., Matsuda, K., et al. 2004, ApJ, 604, 357

Izumiura, H. 1999, in Observational Astrophysics in Asia and its Future, ed. P. S. Chen, Kumming, 77

Kingdon, J., \& Ferland, G. J. 1995, ApJ, 442, 714

Lee, H.-W. 2000, ApJ, 541, L25

Lee, H.-W., \& Hyung, S. 2000, ApJ, 530, L49

Menzies, J. W., Coulson, I. M., Caldwell, J. R. A., \& Corben, P. M. 1982, MNRAS, 200, 463

Mikolajewska, J., \& Kenyon, S. J. 1996, AJ, 112, 1659

Mjalkovskij, M. 1977, Peremenye Zvezdy, 3, 71
Moos, H. W., et al. 2000, ApJ, 538, L1

Munari, U., \& Buson, L. M. 1992, A\&A, 255, 158

Mürset, U., \& Schmid, H. M. 1999, A\&AS, 137, 473

Mürset, U., Nussbaumer, H., Schmid, H. M., \& Vogel, M. 1991, A\&A, 248, 458

Nussbaumer, H., \& Vogel, M. 1989, A\&A, 213, 137

Nussbaumer, H., \& Walder, R. 1993, A\&A, 278, 209

Nussbaumer, H., Schmid, H. M., \& Vogel, M. 1989, A\&A, 211, L27

Payne-Gaposchkin, C. 1946, ApJ, 104, 362

Proga, D., Mikolajewska, J., \& Kenyon, S. J. 1994, MNRAS, 268, 213

Rogerson, J. B., \& Ewell, M. W. 1984, ApJS, 58, 265

Romano, G. 1960, Mem. Soc. Astron. Ital., 31, 11

Sahnow, D. J., Moos, H. W., Ake, T. B., et al. 2000, ApJ, 538, 7

Schmid, H. M. 1989a, in Physics of Classical Novae, ed. A. Cassatella \& R.

Viotti (Berlin: Springer-Verlag), 303

Schmid, H. M. 1989b, A\&A, 211, L31

Schmid, H. M. 1996, MNRAS, 282, 511

Schmid, H. M. 1998, Rev. Mod. Astron., 11, 297

Schmid, H. M., Krautter, J., Appenzeller, I., et al. 1999, A\&A, 348, 950

Seaquist, E. R., Taylor, A. R., \& Button, S. 1984, ApJ, 284, 202 (STB)

Skopal, A. 1998, A\&A, 338, 599

Skopal, A. 2001, A\&A, 366, 157

Skopal, A. 2003a, A\&A, 401, L17

Skopal, A. 2003b, Balt. Astron., 12, 604

Skopal, A. 2005, A\&A, 440, 995

Skopal, A. 2006, A\&A, submitted

Skopal, A., Chochol, D., Pribulla, T., \& Vaňko, M. 2000, IBVS, 5005

Skopal, A., Teodorani, M., Errico, L., et al. 2001, A\&A, 367, 199

Skopal, A., Bode, M. F., Crocker, M. M., et al. 2002a, MNRAS, 335, 1109

Skopal, A., Vaňko, M., Pribulla, T., et al. 2002b, Contrib. Astron. Obs. Skalnaté Pleso, 32, 62 [arXiv: astro-ph/0202249]

Skopal, A., Pribulla, T., Vaňko, M., et al. 2004, Contrib. Astron. Obs. Skalnaté Pleso, 34, 45 [arXiv: astro-ph/0402141]

Skopal, A., Otsuka, M., Tamura, S., et al. 2006, in 7th Pacific Rim Conference on Stellar Astrophysics, ed. Y. W. Kang, \& H.-W. Lee, ASP Conf. Ser., submitted Smits, D. P. 1996, MNRAS, 278, 683

Sokoloski, J. L., Kenyon, S. J., Espey, B. R., et al. 2006, ApJ, 636, 1002

Tomov, N. A., Tomova, M. T., \& Taranova, O. G. 2005, in The Astrophysics of Cataclysmic Variables and Related Objects, ed. J. M. Hameury, \& J. P. Lasota, ASP Conf. Ser., 330, 465

Vlasyuk, V. V. 1993, Bull. Special Astrophys. Obs., 36, 107

Walder, R. 1995, in Wolf-Rayet Stars: Binaries, Colliding Winds, Evolution, ed. K. A. van der Hucht, \& P. M. Williams, IAU Symp., 163, 420

Wallerstein, G., Willson, L. A., Salzer, J., \& Brugel, E. 1984, A\&A, 133, 137

Young, P. R., Dupree, A. K., Espey, B. R., Kenyon, S. J., \& Ake, T. B. 2005, ApJ, 618, 891 
A. Skopal et al.: Structure of the hot object in the symbiotic prototype Z Andromedae during its 2000-03 active phase, Online Material p 1

\section{Online Material}


Table A.1. Observed fluxes of He I emission lines.

\begin{tabular}{cccccc}
\hline \hline Date & JD 2 4... & $F(5876)$ & $\begin{array}{c}F(6678) \\
{\left[\mathrm{erg} \mathrm{cm}^{-2} \mathrm{~s}^{-1}\right]}\end{array}$ & $F(7065)$ & $\mathrm{A} / \mathrm{Q}^{\dagger}$ \\
\hline $10 / 12 / 00$ & 51889.4 & 76.7 & 60.4 & 36.9 & $\mathrm{~A}$ \\
$11 / 12 / 00$ & 51890.4 & 63.7 & 56.4 & 37.3 & $\mathrm{~A}$ \\
$12 / 12 / 00$ & 51891.3 & 77.4 & 57.2 & 33.1 & $\mathrm{~A}$ \\
$30 / 09 / 82$ & $45213^{\ddagger}$ & 19 & 19 & 18 & $\mathrm{Q}$ \\
$01 / 06 / 85$ & $46218^{\ddagger}$ & 28 & 19 & 25 & $\mathrm{~A}$ \\
$02 / 12 / 85$ & $46402^{\ddagger}$ & 58 & 33 & 56 & $\mathrm{~A}$ \\
$20 / 10 / 86$ & $46724^{\ddagger}$ & 39 & 26 & 38 & $\mathrm{~A}$ \\
$01 / 12 / 88$ & $47497^{\ddagger}$ & 15 & 15 & 16 & $\mathrm{Q}$ \\
\hline
\end{tabular}

A/Q: Active/Quiescent phase.

\# From Proga et al. (1994).

\section{Appendix A: He I flux ratios}

To predict flux ratios of He I emission lines, $F(7065) / F(5876)$ and $F(6678) / F(5876)$, Proga et al. (1994) solved the statistical equilibrium equation for a given level in their (19 level) He I atom including the radiative and three-body recombination and effects of collisional ionization and collisional transfer between other levels of the model atom. The model requires as input parameters the electron temperature, $T_{\mathrm{e}}$, the electron density, $N_{\mathrm{e}}$, the rate of photons capable of ionizing $\mathrm{He}$ I atom and the optical depth in the He I $\lambda 3889$ line, $\tau_{3889}$, as a measure of the population of the metastable $2^{3} S$ level. Modeled and observed ratios are shown in Fig. A.1. The location of the flux ratios from the optical maximum in 2000 December suggests that the $\mathrm{He}+$ emitting region is very dense, $N_{\mathrm{e}} \gtrsim 10^{12} \mathrm{~cm}^{-3}$, and radiates at $T_{\mathrm{e}} \gtrsim 20000 \mathrm{~K}$. Comparing the observed luminosity in the $\lambda 5876$ line, $4 \pi d^{2} F(5876)$, with that predicted by its emissivity, $N_{\mathrm{e}}^{2} \varepsilon_{5876} V$, we can determine the effective radius of a spherical nebula as

$R_{\mathrm{eff}}(\mathrm{He}+)=\left(\frac{3 F(5876) d^{2}}{N_{\mathrm{e}}^{2} \varepsilon_{5876}}\right)^{1 / 3}$

where the emission coefficient $\varepsilon_{5876} \doteq 1 \times 10^{-26} \mathrm{erg} \mathrm{cm}^{3} \mathrm{~s}^{-1}$ for $N_{\mathrm{e}}=10^{12} \mathrm{~cm}^{-3}$ and $\tau_{3889}=100$ (Proga et al. 1994). Then $F(5876)=73 \times 10^{-13} \mathrm{erg} \mathrm{cm}^{-2} \mathrm{~s}^{-1}$ (Table A.1), and $d=1.5 \mathrm{kpc}$ yield $R_{\text {eff }}(\mathrm{He}+) \doteq 5 R_{\odot}$.

During the recent major outburst He I fluxes evolved toward lower $F(7065) / F(5876)$, whereas during the smaller 1986eruption the fluxes evolved toward lower $F(6678) / F(5876)$ at nearly constant $F(7065) / F(5876)$ (Fig. A.1). In general, this reflects different properties of the nebula, which develops during outbursts around the hot active object. Qualitatively, during strong outbursts the velocity field of the hot star wind can make large differences in the density throughout the nebula. As a result, contributions from the outer, low-density part of the nebula will enhance more the $\lambda 5876$ line relative to other lines and thus lower the considered flux ratios. According to Smits (1996) the relative fluxes $F(5876) / F(6678) / F(7065)=2.519 / 0.682 / 0.637$ for $N_{\mathrm{e}}=10^{6} \mathrm{~cm}^{-3}$ and $T_{\mathrm{e}}=20000 \mathrm{~K}$ (open circle in Fig. A.1). Therefore the real size of the $\mathrm{He}+$ nebula will probably exceed the effective radius derived for a constant density distribution. In addition, its shape is not spherical due to the presence of the optically thick disk-like matter at the orbital plane, which prevents ionization in these directions (Sect. 4).

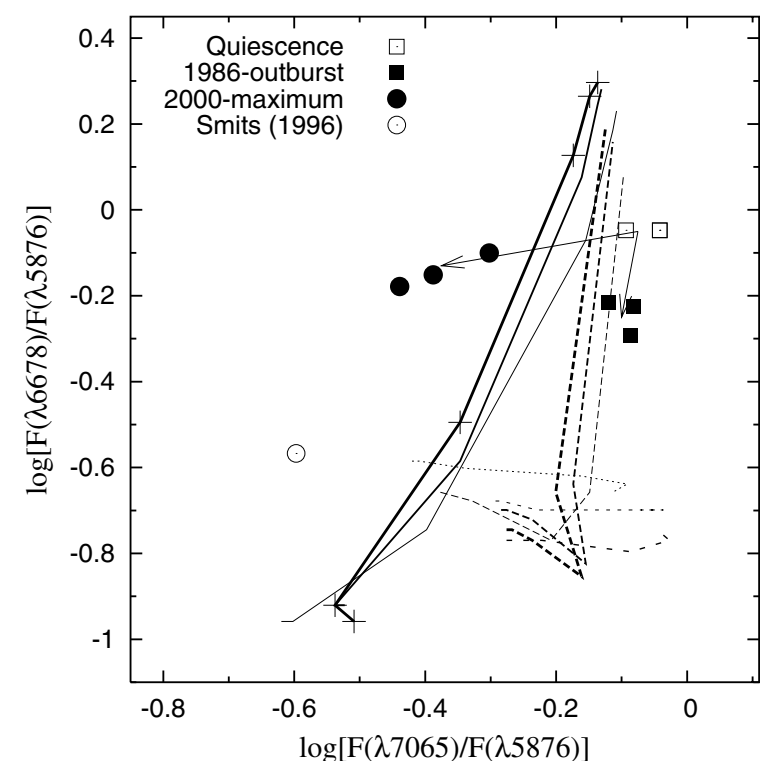

Fig. A.1. Dependencies of He I flux ratios, $F(7065) / F(5876)$ and $F(6678) / F(5876)$, for $N_{\mathrm{e}}=10^{6} \mathrm{~cm}^{-3}$ (dotted lines), $N_{\mathrm{e}}=10^{10} \mathrm{~cm}^{-3}$ (dashed lines) and $N_{\mathrm{e}}=10^{12} \mathrm{~cm}^{-3}$ (solid lines), for $T_{\mathrm{e}}=10000 \mathrm{~K}$, $15000 \mathrm{~K}$ and $20000 \mathrm{~K}$ (light, medium and heavy lines, respectively). Crosses indicate $\tau_{3889}=0.01,0.1,1,10,100,1000$ from the bottom to the top on the solid heavy line (Table 5 of Proga et al. 1994). Observed fluxes are from Table A.1. The open circle corresponds to a low-density nebula $\left(N_{\mathrm{e}}=10^{6} \mathrm{~cm}^{-3}, T_{\mathrm{e}}=20000 \mathrm{~K}\right)$.

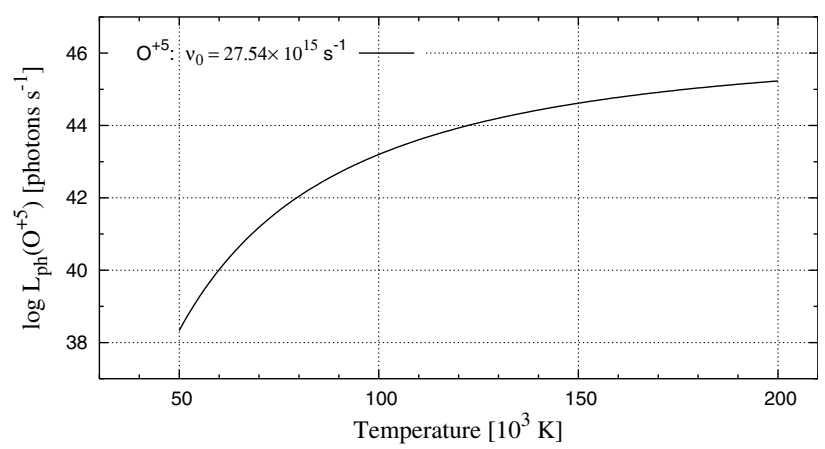

Fig. B.1. Number of photons capable of ionizing $\mathrm{OV}$ ions as a function of temperature scaled to the luminosity of $1000 L_{\odot}$.

\section{Appendix B: Radius of the O VI zone}

The radius of the $\mathrm{OVI}$ zone, $R(\mathrm{OVI})$, can be obtained from the equation of the equilibrium between the flux of photons, $L_{\mathrm{ph}}(\mathrm{OV})$, capable of ionizing $\mathrm{OV}$ ions and the number of recombinations by $\mathrm{O}$ VI ions. For a spherically symmetric nebular medium around the central ionizing source we can write

$L_{\mathrm{ph}}(\mathrm{O} \mathrm{v})=\frac{4 \pi}{3} R\left(\mathrm{O} \mathrm{VI}^{3} A(\mathrm{O} \mathrm{VI}) N_{+} N_{\mathrm{e}} \alpha_{\mathrm{B}}(\mathrm{O} \mathrm{VI})\right.$,

where $A(\mathrm{OVI})$ is the abundance of $\mathrm{OVI}$ ions, $\alpha_{\mathrm{B}}(\mathrm{OVI})$ is the recombination coefficient of a free electron with the $\mathrm{O}$ VI ion for the case $B$ and $N_{+}$and $N_{\mathrm{e}}$ are concentrations of protons and electrons. In determining $L_{\mathrm{ph}}(\mathrm{OV})$ values we integrated the Plank curve from the ionization limit of $113.896 \mathrm{eV}\left(v_{0}=\right.$ $\left.27.54 \times 10^{15} \mathrm{~s}^{-1}\right)$ to $\infty$. Figure B. 1 shows the $L_{\mathrm{ph}}(\mathrm{O} \mathrm{v})$ quantity as a function of the temperature for a luminosity of the ionizing source of $1000 L_{\odot}$. Further we assumed that all oxygen atoms are ionized to $\mathrm{O}$ VI within the zone, i.e. $A(\mathrm{O} V \mathrm{I})=A(\mathrm{O} \mathrm{I})$, for which we adopted the solar photospheric abundance of $4.6 \times 10^{-4}$ 
A. Skopal et al.: Structure of the hot object in the symbiotic prototype Z Andromedae during its 2000-03 active phase, Online Material $p 3$

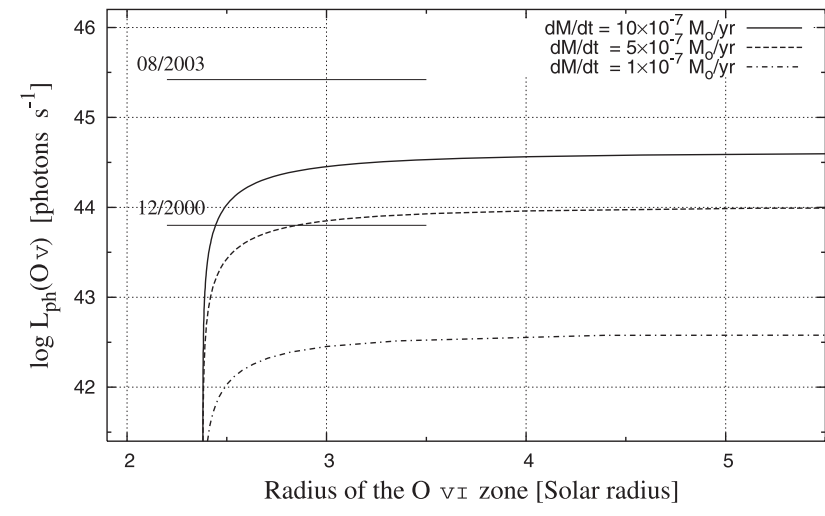

Fig. B.2. Radius of the O VI zone as a function of $L_{\mathrm{ph}}(\mathrm{OV})$ photons for particle distribution in a form of the stellar wind from the ionizing source (Eq. (B.4)). Horizontal bars indicate quantities from the optical maximum (2000 December) and the 2003 post-outburst stage.

(Asplund et al. 2004) and $\alpha_{\mathrm{B}}(\mathrm{O} \mathrm{VI})=9.5 \times 10^{-12} \mathrm{~cm}^{+3} \mathrm{~s}^{-1}$ for $T_{\mathrm{e}}=30000 \mathrm{~K}$ (Gurzadyan 1997). According to Sokoloski et al. (2006), the temperature of the ionizing source $T_{\mathrm{h}}^{\text {i.s. }}=$ $95000 \mathrm{~K}$ and $160000 \mathrm{~K}$ during the optical maximum and the 2003 post-outburst stage, which implies $L_{\mathrm{ph}}(\mathrm{O} \mathrm{v})=6.4 \times 10^{43}$ and $2.6 \times 10^{45}$ photons s $^{-1}$ for luminosities of 7000 and $4400 L_{\odot}$, respectively (Fig. B.1, Table 2). For a particle concentration of the order of $10^{12} \mathrm{~cm}^{-3}$, which can be expected in the close vicinity of the ionizing source due to the mass loss via the stellar wind at rates of $\sim 10^{-6} M_{\odot} \mathrm{yr}^{-1}$ (Skopal et al. 2006), one can derive

$R(\mathrm{OVI}) \approx \mathrm{a}$ few $\times R_{\odot}$

during the optical maximum. In a more rigorous approach we integrate densities of the hot star wind from a radius $r_{1}$ above its origin, $R_{\mathrm{w}}$, to the end of the $\mathrm{O}$ VI zone (i.e. the $R(\mathrm{O} \mathrm{VI})$ radius). Then Eq. (B.1) takes the form

$L_{\mathrm{ph}}(\mathrm{O} v)=4 \pi \alpha_{\mathrm{B}}(\mathrm{O} \mathrm{VI}) A(\mathrm{O} \mathrm{vI}) \int_{r 1}^{R(\mathrm{OVI})} N(r)^{2} r^{2} \mathrm{~d} r$, where $N(r)=\dot{M} / 4 \pi r^{2} \mu m_{\mathrm{H}} v(r)$ is determined via the continuous mass loss rate $\dot{M}$ from the hot star with the velocity field $v(r)=$ $v_{\infty}\left(1-R_{\mathrm{w}} / r\right)^{\beta}$ (Castor et al. 1975). This allows us to express the last equation as

$L_{\mathrm{ph}}(\mathrm{O} \mathrm{v})=\frac{\alpha_{\mathrm{B}}(\mathrm{O} \mathrm{VI}) A(\mathrm{O} \mathrm{vI})}{4 \pi\left(\mu m_{\mathrm{H}}\right)^{2}}\left(\frac{\dot{M}}{v_{\infty}}\right)^{2} \frac{1}{R_{\mathrm{w}}} \times I$,

where the integral

$I=\frac{1}{(2 \beta-1)}\left[\left(1-\frac{R_{\mathrm{W}}}{r_{1}}\right)^{1-2 \beta}-\left(1-\frac{R_{\mathrm{w}}}{R(\mathrm{O} \mathrm{VI})}\right)^{1-2 \beta}\right]$.

A solution of Eq. (B.4) is shown in Fig. B.2 for three different $\dot{M}$. Parameters $\beta=1.7, v_{\infty}=2000 \mathrm{~km} \mathrm{~s}^{-1}, R_{\mathrm{w}}=1.7 R_{\odot}$ and $r_{1} \sim 1.4 R_{\mathrm{w}}$ were estimated from modeling the broad $\mathrm{H} \alpha$ wings during the maximum (Skopal et al. 2006). Also in this case the $R\left(\mathrm{O}\right.$ VI) radius can be small, $<3 R_{\odot}$, for mass-loss rates between $1 \times 10^{-6}$ and $5 \times 10^{-7} M_{\odot} \mathrm{yr}^{-1}$ and the observed flux of ionizing photons $L_{\mathrm{ph}}(\mathrm{OV})=6.4 \times 10^{43} \mathrm{~s}^{-1}$. However, for $L_{\mathrm{ph}}(\mathrm{OV}) \gtrsim 10^{44}-10^{45} \mathrm{~s}^{-1}$ the O VI zone can expand to infinity. This is in qualitative agreement with the observed significant and fast increase in the O VI $\lambda 1032,1038$ fluxes from the optical maximum (2000 December) to the next FUSE observation in 2001 July (Table 3), which thus can be understood as a result of the increase in $T_{\text {h.s. }}^{\text {i.s. }}$ to $120000-150000 \mathrm{~K}$ at approximately constant luminosity (Sokoloski et al. 2006). 